\title{
Research Paper \\ Comparison of the Effectiveness of Music Therapy and Cognitive Behavioral Thera- py on Quality of Life, Craving and Emotion Regulation in Patients Under Methadone Maintenance Therapy
}

\section{Seyed Reza Eshaghi Farahmand ${ }^{1}(1),{ }^{*}$ Hassan Ahadi $^{2}{ }^{\circ}$, Maryam Kalhornia Golkar ${ }^{3}$, Mastoreh Sedaghat ${ }^{4}$}

\author{
1. Department of Health Psychology, Kish International Branch, Islamic Azad University, Kish Island, Iran \\ 2. Department of Psychology, Allameh Tabataba'i University, Tehran, Iran. \\ 3. Department of Psychology, Karaj Branch, Islamic Azad University, Karaj, Iran. \\ 4. Department of Psychology, Semnan Branch, Islamic Azad University, Semnan, Iran
}

\begin{tabular}{|c|c|}
\hline $\begin{array}{l}\text { Use your device to scan } \\
\text { and read the article online }\end{array}$ & Cftation Eshaghi Farahmand S, Ahadi H, Kalhornia Golkar M, Sedaghat M. [Comparison of the Effectiveness of Music \\
\hline 口iciti & and Cognitive Behavioral Therapy on Quality of Life, Craving and Emotion Regulation in Patients Under Methadone \\
\hline 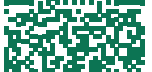 & $\begin{array}{l}\text { Maintenance Therapy (Persian)]. Iranian Journal of Psychiatry and Clinical Psychology. 2020; 26(2):170-187. http://dx.doi. } \\
\text { org/10.32598/ijpcp.26.2.3212.1 }\end{array}$ \\
\hline 口ifat & dol'http://dx.doi.org/10.32598/ijpcp.26.2.3212.1 \\
\hline
\end{tabular}

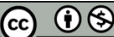

Received: 28 Sep 2019

Accepted: 07 Jan 2020

Available Online: $01 \mathrm{Jul} 2020$

Key words:

Music therapy,

Cognitive behavioral

therapy, Quality of Life

(QoL), Emotion regulation, Craving

\section{A B STRACT}

Objectives The purpose of this study was to compare the effectiveness of Music Therapy and Cognitive Behavioral Therapy on Quality of Life (QoL), craving, and emotional regulation in patients under methadone maintenance therapy.

Methods The method of the study was quasi-experimental and multi-group pre-test and post-test design. The statistical population consisted of all clients treated with methadone maintenance therapy in addiction treatment clinics affiliated with Tehran Health Organization in 2018. Then 54 people of whom (18 in each group) were selected through purposeful sampling in two experimental groups (Music-Therapy and Cognitive Behavioral Therapy) and a control group was included. The data was collected using the Cognitive Emotion Regulation Questionnaire, the Quality of Life Questionnaire, and Craving Questionnaire. The ANCOVA was used for data analysis.

Results The results showed that both Music Therapy and Cognitive Behavioral Therapy Improved the QoL, emotion regulation in the experimental group compared to the control group and significantly reduced craving $(\mathrm{P}<0.01)$. The results of the follow-up test showed that the mean scores of the experimental groups in the study variables were significantly different from the control group.

Conclusion There was a significant difference between the treatment groups in terms of their effect on the QoL and negative cognitive emotion regulation so that Cognitive Behavioral Therapy was more effective in regulating negative emotions and enhancing the QoL but there was no significant difference in regulating for positive emotions and reducing craving. These results suggest that both treatments significantly increase the QoL, emotional regulation, and it reduces craving.

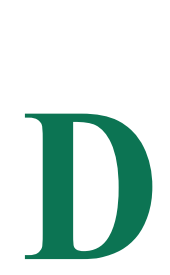

\section{Extended Abstract}

\section{Introduction}

ue to the widespread use of methadone maintenance therapy in Iran and a $48.2 \%$ relapse rate in this treatment [31], as well as the poor treatment with no psychosocial interventions on treatment compliance, and among the high dropouts. The need to use other psychological therapies associated with this treatment, including standard Cognitive Behavioral Therapy to reduce craving [21], to increase QoL [29], and

-

* Corresponding Author:

Hassan Ahadi, PhD.

Address: Department of Psychology, Allameh Tabataba'i University, Tehran, Iran.

Tel: +98 (912) 6049225

E-mail:drhahadi5@gmail.com 
the Emotional Regulation of Ford, et al. [32] is felt over and over. On the other hand, while Cognitive Behavioral Therapy seeks to directly change the behavior of people with substance abuse, Music Therapy has positive effects on mood and by reducing their negative emotions. Numerous studies have also shown that Cognitive Behavioral Therapy has no advantage over other treatments that are not focused on coping skills [33]. Therefore, the question of the present study is whether Cognitive Behavioral Therapy as a standard treatment of Music Therapy as a creative and complementary treatment of addiction is different in QoL, craving, and emotional regulation of methadone maintenance patients or not.

\section{Methods}

The method of this study was semi-experimental with pre-test and post-test. The statistical population of this study consisted of all clients undergoing methadone maintenance therapy in addiction treatment clinics affiliated to Tehran Health Organization in 2019. In this study, first, in coordination with the Health Care System of Tehran, referring to addiction clinics, they were asked to refer to methadone maintenance practitioners who have at least one year of treatment experience.

The inclusion criteria for this study were as follows: addicts on methadone maintenance treatment, male gender, no physical problems, no history of exposure to psychological training, age 18-40 years, no history of drug use.

The exclusion criteria were as follows: failure of the sample group or family to participate in each phase of the study, having physical and mental problems, history of psychiatric problems, professional athletes, over 40 years of age, and drug use.

The researcher interviewed and surveyed the inclusion and exclusion criteria and 60 persons were selected by purposive sampling; then, randomly divided into three groups. The researcher then randomly selected into two groups as the experimental group and control group. Then one of the experimental groups was randomly assigned to 8 sessions of Music Therapy group and the other group 8 sessions of Cognitive Behavioral Therapy by the researcher. The control group received no training. Data were collected by using the Cognitive Emotion Regulation Questionnaire [34], QoL Questionnaire [36], and Craving Questionnaire [38]. The ANCOVA method was used for data analysis.

\section{Results}

The purpose of this study was to compare the efficacy of Music Therapy and cognitive behavioral therapy on QoL, craving, and emotional regulation in methadone maintenance patients. The findings showed that Music Therapy increased QoL, negative cognitive emotion and positive emotions and decreased craving. Also, findings showed that cognitive behavioral therapy increased QoL and regulated positive emotions and decreased craving. Negative emotions were regulated (Tables 1-5).

Table 1. Mean $\pm S D$ of Variables in the experimental and control groups

\begin{tabular}{cccc}
\hline \multirow{2}{*}{ Variables } & Groups & \multicolumn{2}{c}{ Mean \pm SD } \\
\cline { 2 - 4 } & & Pre-test & Post-test \\
\hline \multirow{2}{*}{ Quality of Life } & Cognitive Behavioral Therapy & $104.27 \pm 8.89$ & $124.50 \pm 6.87$ \\
& Music Therapy & $103.83 \pm 15.22$ & $112.05 \pm 13.26$ \\
Positive cognitive emotion regulation & Control & $108.05 \pm 7.24$ & $109.22 \pm \pm 7.71$ \\
& Cognitive Behavioral Therapy & $48 \pm 9.12$ & $61.98 \pm 9.02$ \\
& Music Therapy & $51.61 \pm 8.56$ & $57.00 \pm 9.36$ \\
Negative cognitive emotion regulation & Control & $53.83 \pm 10.66$ & $50.22 \pm 5.51$ \\
& Cognitive Behavioral Therapy & $58.38 \pm 12.30$ & $47.83 \pm 7.88$ \\
& Music Therapy & $59.72 \pm 9.89$ & $50.27 \pm 6.28$ \\
Craving & Control & $55.84 \pm 8.04$ & $56.88 \pm 4.94$ \\
& Cognitive Behavioral Therapy & $72.50 \pm 12.06$ & $49.77 \pm 7.68$ \\
& Music Therapy & $75.70 \pm 13.02$ & $55.77 \pm 10.45$ \\
& Control & $71.61 \pm 9.46$ & $69.27 \pm 7.25$ \\
\hline
\end{tabular}


Table 2. Results of test of Levinss variance homogeneity and slope homogeneity in experimental and control group based on research variables

\begin{tabular}{|c|c|c|c|c|c|c|c|c|}
\hline \multirow{2}{*}{ Variable } & \multicolumn{2}{|c|}{$\begin{array}{l}\text { Levin's Variance Homo- } \\
\text { geneity }\end{array}$} & \multicolumn{2}{|c|}{ slope Homogeneity } & \multicolumn{2}{|c|}{ Linearity } & \multicolumn{2}{|c|}{$\begin{array}{c}\text { The Distribution of Data is } \\
\text { Normal }\end{array}$} \\
\hline & $\mathbf{F}$ & $\mathbf{P}$ & $\mathbf{F}$ & $\mathbf{P}$ & $\mathbf{F}$ & $\mathbf{P}$ & K-S & $\mathbf{P}$ \\
\hline Quality of Life & 1.12 & 0.33 & 1.36 & 0.28 & 61.45 & 0.001 & 0.69 & 0.713 \\
\hline $\begin{array}{l}\text { Positive cognitive emo- } \\
\text { tion regulation }\end{array}$ & 2.22 & 0.12 & 1.61 & 0.20 & 22.83 & 0.001 & 0.87 & 0.593 \\
\hline $\begin{array}{l}\text { Negative cognitive emo- } \\
\text { tion regulation }\end{array}$ & 1.22 & 0.30 & 1.10 & 0.38 & 4.86 & 0.04 & 0.16 & 1.120 \\
\hline Craving & 1.25 & 0.29 & 21.07 & 0.001 & 91.67 & 0.001 & 0.63 & 0.748 \\
\hline
\end{tabular}

Table 3. One-way analysis of covariance of Quality of Life in two experimental and one control group

\begin{tabular}{|ccccccc}
\hline The Dependent Variable & $\begin{array}{c}\text { The Sum of the } \\
\text { Squares }\end{array}$ & df & Mean Squares & F & Sig. & Eta Squared \\
\hline Quality of Life & 46.2441 & 2 & 1220.73 & 45.37 & $\leq 0.001$ & 0.67 \\
\hline Positive cognitive emotion regulation & 1787.62 & 2 & 893.81 & 30.61 & $\leq 0.001$ & 0.59 \\
\hline Negative cognitive emotion regulation & 1169.42 & 2 & 584.71 & 22.73 & $\leq 0.001$ & 0.52 \\
\hline Craving (Post-test difference pre-test) & 7697.65 & 2 & 3848.82 & 74.49 & $\leq 0.001$ & 0.74 \\
\hline
\end{tabular}

Table 4. Follow-up test results to compare groups` means of Quality of Life in the post-test phase

\begin{tabular}{|c|c|c|c|c|c|}
\hline Scale & Comparison & $\begin{array}{l}\text { Average Differ- } \\
\text { ence }\end{array}$ & $\begin{array}{l}\text { The Standard } \\
\text { Error }\end{array}$ & Sig. & Eta Squared \\
\hline \multirow{3}{*}{ Quality of Life } & Group 1 \& 2 & 11.57 & 1.85 & $\leq 0.001$ & \multirow{3}{*}{0.67} \\
\hline & Group 1 \& 3 & 17.67 & 1.87 & $\leq 0.001$ & \\
\hline & Group 2 \& 3 & 6.10 & 1.80 & $=0.005$ & \\
\hline \multirow{3}{*}{$\begin{array}{l}\text { Positive cognitive emotion } \\
\text { regulation }\end{array}$} & Group $1 \& 2$ & 8.64 & 3.80 & $=0.08$ & \multirow{3}{*}{0.59} \\
\hline & Group $1 \& 3$ & 16.47 & 2.43 & $\leq 0.001$ & \\
\hline & Group $2 \& 3$ & 7.82 & 2.61 & $=0.01$ & \\
\hline \multirow{3}{*}{$\begin{array}{l}\text { Negative cognitive emotion } \\
\text { regulation }\end{array}$} & Group 1\&2 & -11.65 & 3.59 & $=0.007$ & \multirow{3}{*}{0.52} \\
\hline & Group $1 \& 3$ & -14.64 & 2.28 & $\leq 0.001$ & \\
\hline & Group $2 \& 3$ & -2.98 & 2.45 & $=0.69$ & \\
\hline \multirow{3}{*}{$\begin{array}{l}\text { (Post-test difference pre- } \\
\text { test) }\end{array}$} & Group $1 \& 2$ & -2.02 & 2.39 & $=0.99$ & \multirow{3}{*}{0.74} \\
\hline & Group $1 \& 3$ & -26.27 & 2.39 & $\leq 0.001$ & \\
\hline & Group 2\&3 & -24.25 & 2.39 & $\leq 0.001$ & \\
\hline
\end{tabular}

Explanation: 1 = Cognitive Behavioral Therapy Group 2 = Music Therapy Group 3 = Control Group

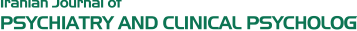


Table 5. Follow-up test results to compare groups` means of Quality of Life in the post-test phase

\begin{tabular}{|c|c|c|c|c|c|}
\hline Scale & $\begin{array}{c}\text { Comparison of } \\
\text { Groups }\end{array}$ & Average Difference & Standard Error & Sig. & Eta squared \\
\hline \multirow{3}{*}{ Quality of Life } & $1 \& 2$ & 11.57 & 1.85 & $P \leq 0.001$ & \multirow{3}{*}{0.67} \\
\hline & $1 \& 3$ & 17.57 & 1.87 & $P \leq 0.001$ & \\
\hline & $2 \& 3$ & 6.10 & 1.80 & $P=0.005$ & \\
\hline \multirow{3}{*}{$\begin{array}{l}\text { Positive cognitive } \\
\text { emotion regulation }\end{array}$} & $1 \& 2$ & 8.64 & 3.80 & - & \multirow{3}{*}{0.59} \\
\hline & $1 \& 3$ & 16.47 & 2.43 & - & \\
\hline & $2 \& 3$ & 7.82 & 2.61 & - & \\
\hline \multirow{3}{*}{$\begin{array}{l}\text { Negative cognitive } \\
\text { emotion regulation }\end{array}$} & $1 \& 2$ & -11.65 & 3.59 & - & \multirow{3}{*}{0.52} \\
\hline & $1 \& 3$ & -14.64 & 2.28 & - & \\
\hline & $2 \& 3$ & -2.98 & 2.45 & - & \\
\hline \multirow{3}{*}{$\begin{array}{l}\text { (Post-test difference } \\
\text { pre-test) }\end{array}$} & $1 \& 2$ & -2.02 & 2.39 & - & \multirow{3}{*}{0.74} \\
\hline & $1 \& 3$ & -26.27 & 2.39 & - & \\
\hline & $2 \& 3$ & -24.25 & 2.39 & - & \\
\hline
\end{tabular}

\section{Discussion}

Comparison of Music Therapy and cognitive behavioral therapy compared with cognitive behavioral therapy showed that Cognitive Behavioral Therapy had an effective role in enhancing $\mathrm{QoL}$ and reducing negative emotions than Music Therapy. Mental Analysis Irvine et al.; Carroll et al.; Kaden; Silva and Serra showed that cognitive-behavioral interventions have a significant role in reducing the use and experience of the patient's experience and play a major role in improving psychological symptoms and reducing the rate of relapse and return to substance use [51]. Also, the results are consistent with the findings of McGaugh, Heron, and Otto [52]; Ocilla, Hepner, Manoz \& Watkins [55], and Driessen \& Hollon [55] who showed that a Cognitive Behavioral Therapy of addiction improves mood and tolerance, communication with others, and QoL. But there was no significant difference concerning Craving and positive cognitive emotion regulation.

As the results also point out, Music Therapy is a complementary therapy and can play an important role along with other therapies such as drug therapy, and it has a stronger role in enhancing positive emotions than negative emotions.
One of the important limitations of this study was the difficulty in satisfying the sample group for cooperation. Also Controlling for other disturbing variables (use of other medications, income, social support) decreased the subjects' ability to control these variables. Caution should be exercised when generalizing the results to the female gender because of the use of the male gender. Finally, it is recommended to use Music Therapy as a complementary therapy.

\section{Ethical Considerations}

\section{Compliance with ethical guidelines}

This study has been registered by the Iranian Registry of Clinical Trials (Code: IRCT20191030045280N1). All ethical principles were observed in this study. Participants were free to leave the study at any time and were assured of the confidentiality of their information

\section{Funding}

This research did not receive any grant from funding agencies in the public, commercial, or non-profit sectors. 


\section{Authors contributions}

All authors were equally contributed in preparing this article.

\section{Conflicts of interest}

The authors declared no conflict of interest. 


\title{
مقايسه اثربخشى موسيقى هرمانى و رفتاردرمانى شناختى بر كيفيت زندكَى، ولع مصرف و تنظيم هيجانى بيماران تحت درمان نتخهدارنده بانيان متادون
}

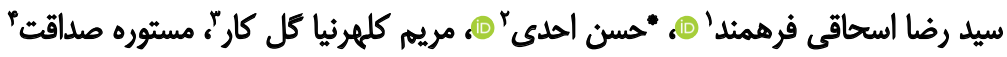 \\ 1. كروه روانشناسى سلامت، واحد بين المللى كيش، دانشكاه آزاد اسلامى، جزيره كيش، ايران.

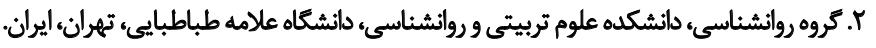

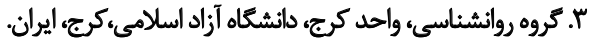

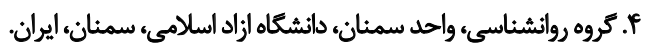

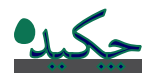

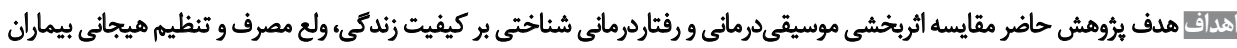

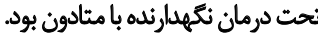

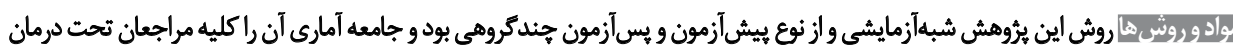

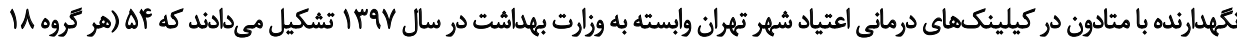

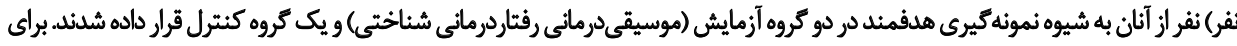

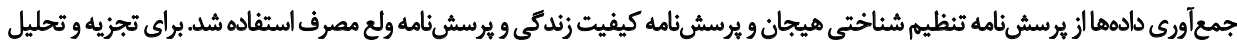

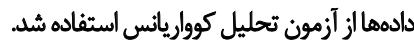

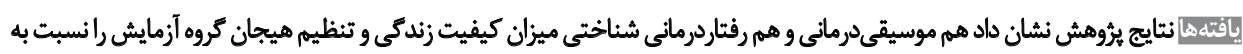

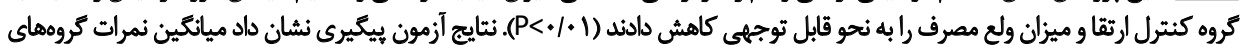

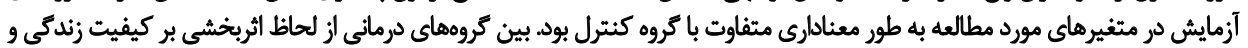

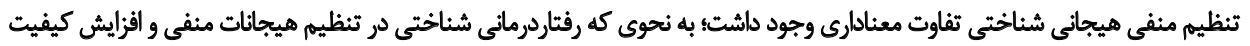

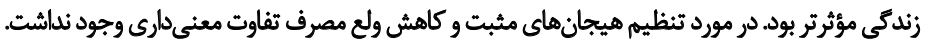

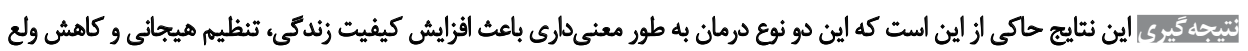
مصرف ميىشود

تاريخ دريافت: 9. مهر 1raג تاريخ هذيرش: VI أدى

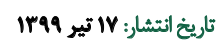

كليدوازوها: موسيقي درماني، رفتاردرمانى

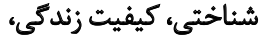
تنظيمي هيجان، ولع مصرفي رنئ
\end{abstract}

اين افراد خدمات حرفهاي رادريافت مي كنند [ـإ] و همجنان نياز

مقدمه

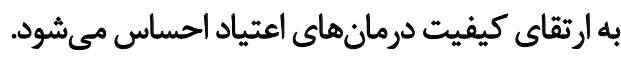

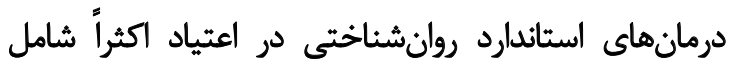

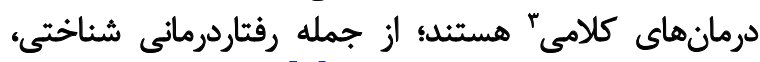

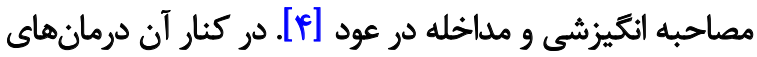

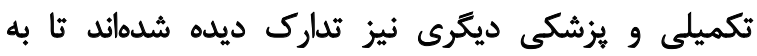

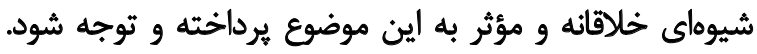

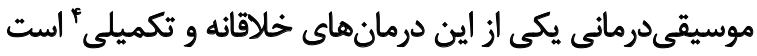

استفاده و سوءاستفاده از مواد و الكل با انواع آسيبهاى

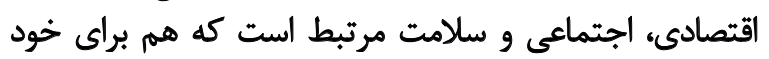

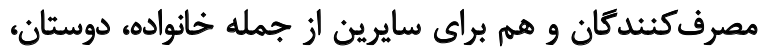

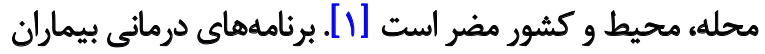

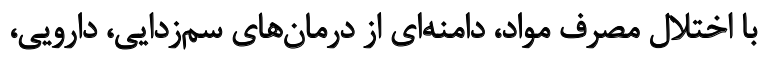

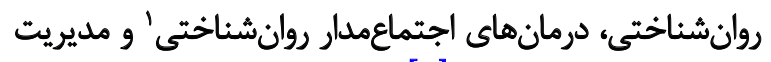

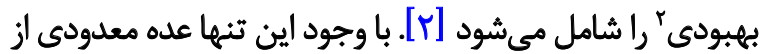


كند [IV] همجينين در بسيارى از مطالعات، موسيقىدرمانى يا

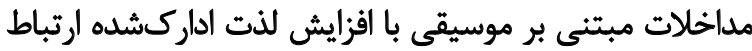

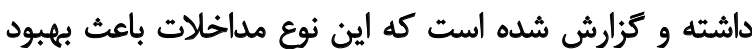

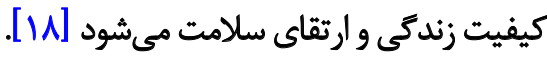
مطالعات معدودى نيز اثر مداخلات مبتنى بر موسيقى را بر

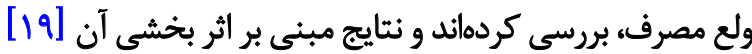

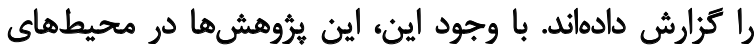

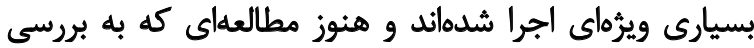

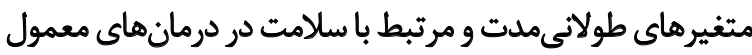

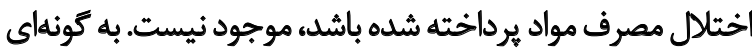

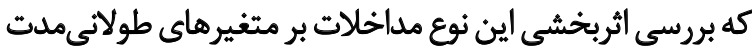

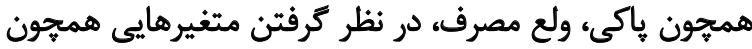

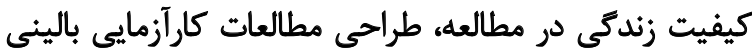

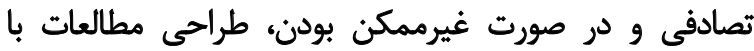

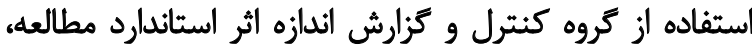

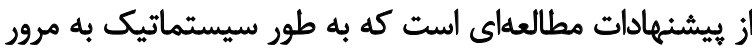

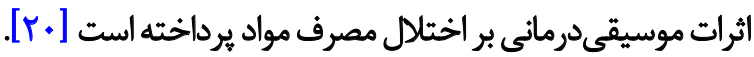

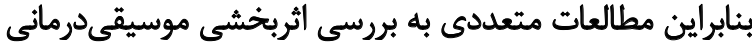

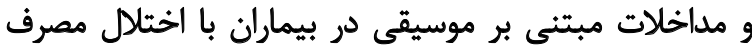

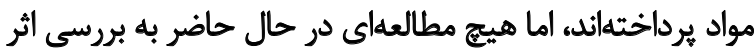

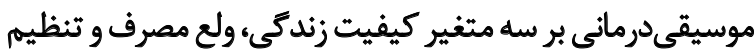

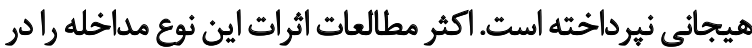

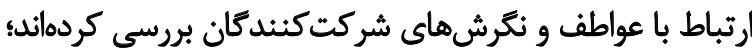

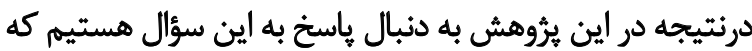

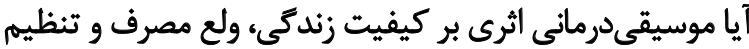

هيجانى بيماران تحت درمان نكَهدارنده با با متادون دارد؟ همجِنين از طرف ديكر رويكرد رفتاردرمانى شناختى به عنوان

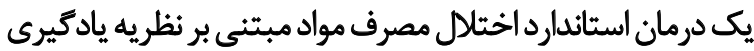

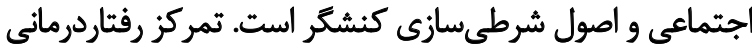

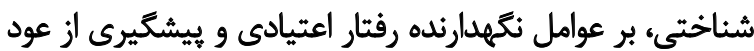

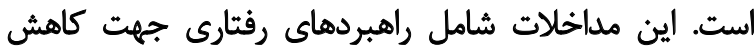

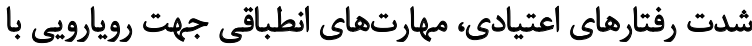

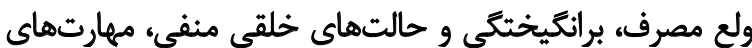

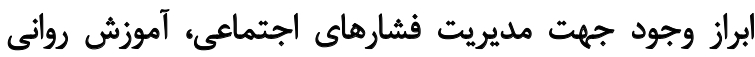

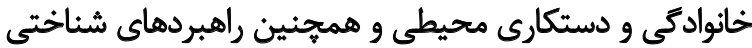

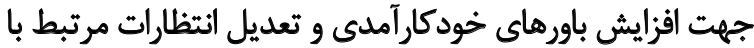

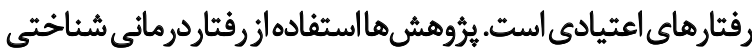

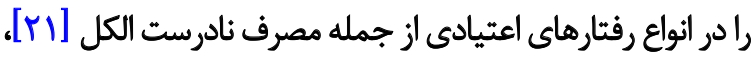

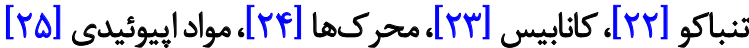

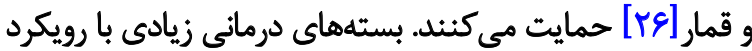

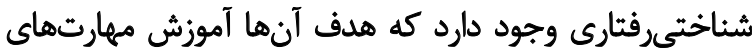

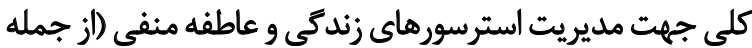

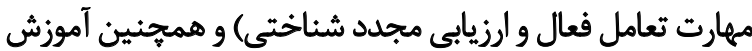

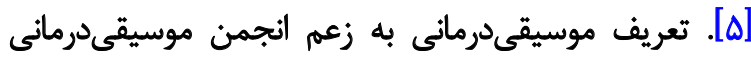

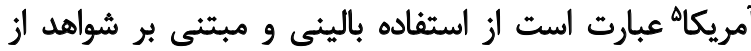

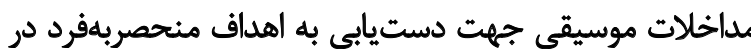

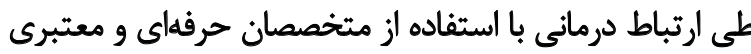
كه دوره موسيقى ارنيانى درمانى را كذراندهاند.

جهت روشن شدن اثرات بالينى موسيقىدرمانى و مداخلات

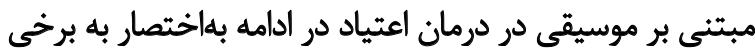

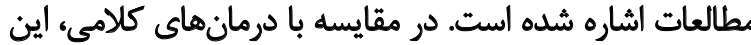
درمان و اين نوع مداخلات فرصت متفاوتى را براى خدوان خودابرازى

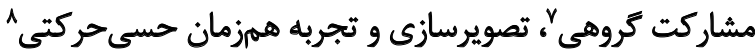

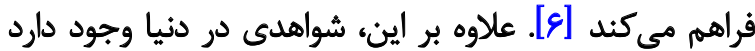

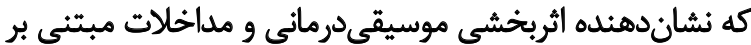

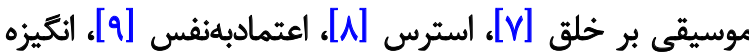

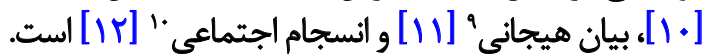

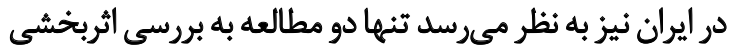

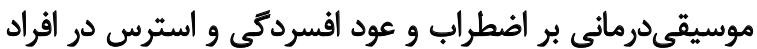

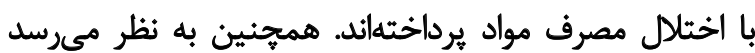

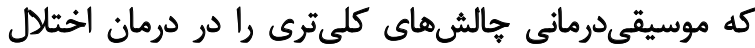

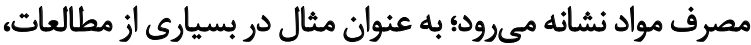

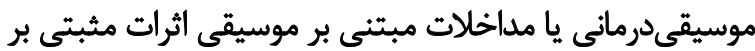

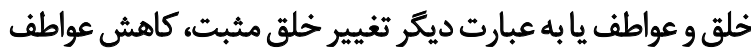

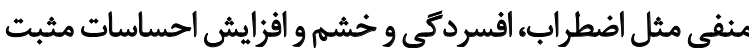

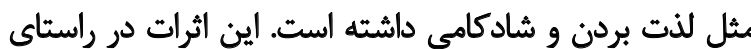

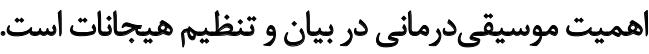

موسيقىدرانى فرصت هايى را براى جستوجو و بيان

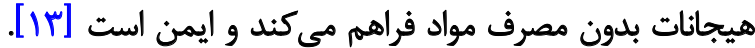

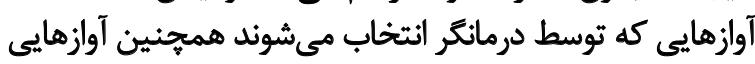

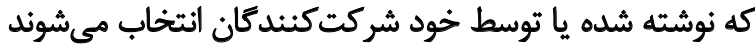

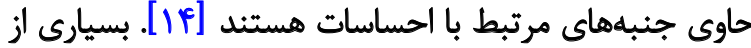

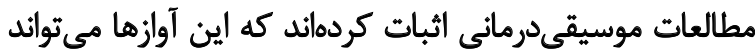

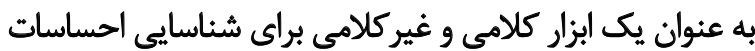

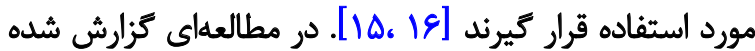

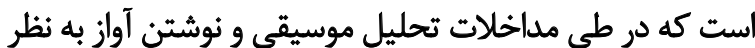

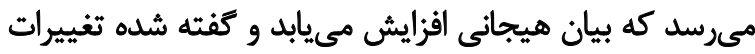

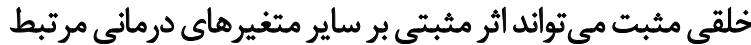

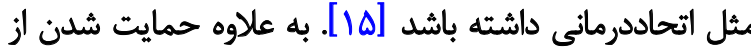

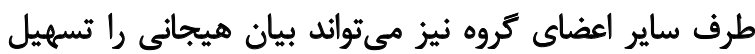

5. American Music Therapy Association

6. Self- Expression

7. Cooperative Group Activity

8. Synchronized Sensorimotor Experience

9. Emotional Expression

10. Social Cohesion 
درمان از جمله درمان استاندارد شناختيرفيتارى جهت كاهش

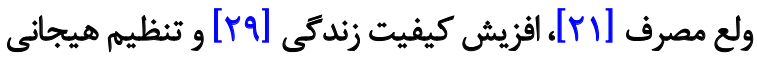

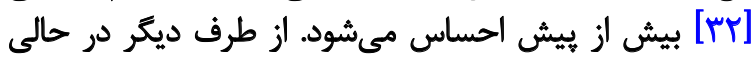

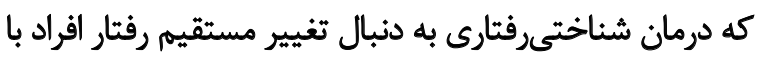

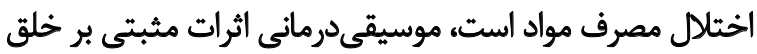

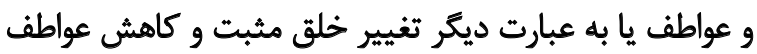

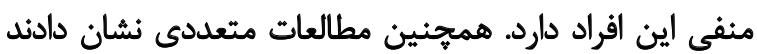

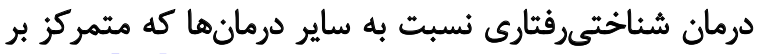

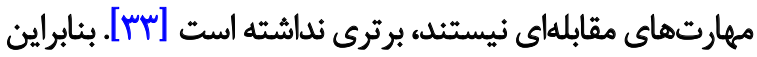

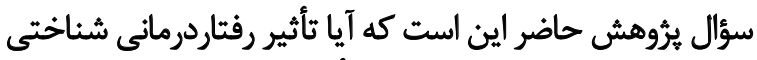

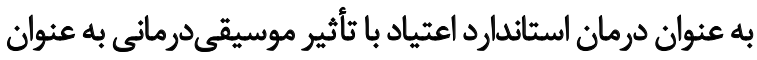

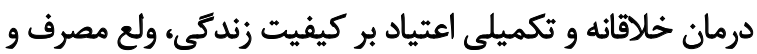

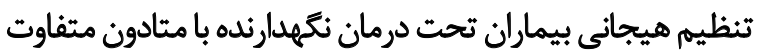

است؟ تنطي

وروش

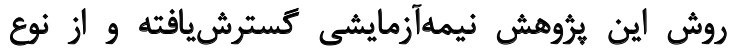

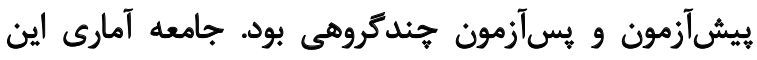

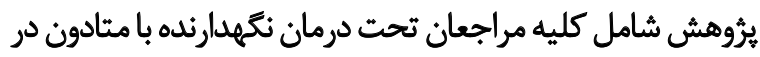

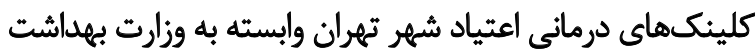

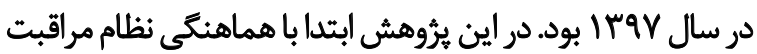

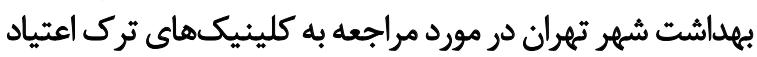

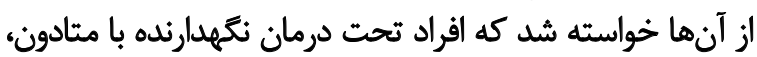

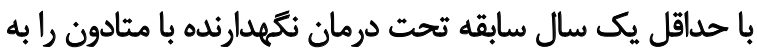

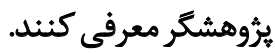

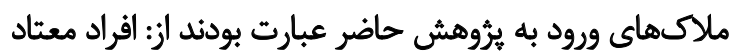

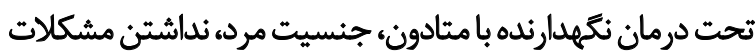

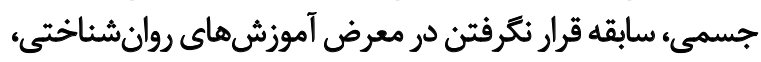

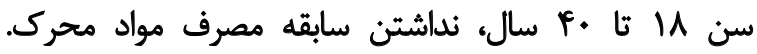

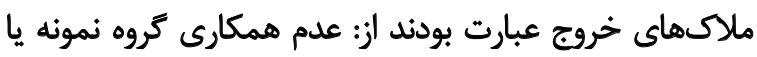

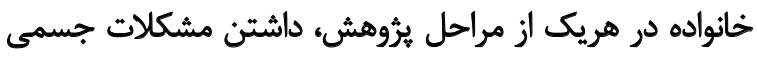

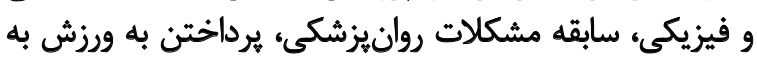
شكل حرفهاى و سن بالاى ·f سال.

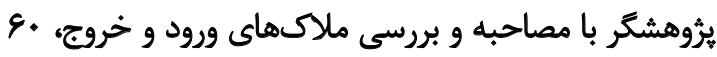

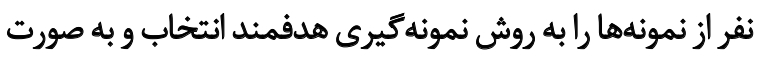

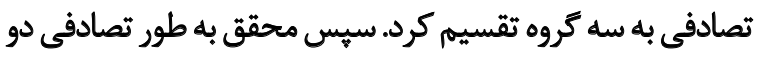

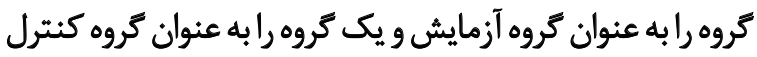

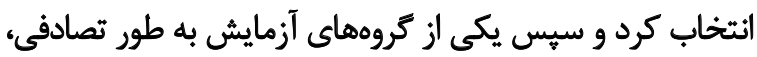

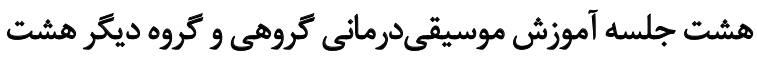

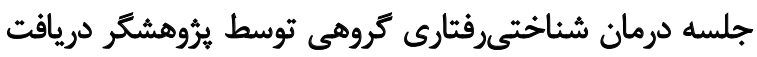
كردند و كروه كنترل آموزشى دريتافي كروهى نكرد.
اختصاصى براى ولع مصرف (مثل رد كردن مصرفه و كنترل

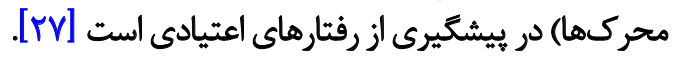
بيشتر يُروهشها در اين نكته توافق دارند كه مداخلات مؤثر

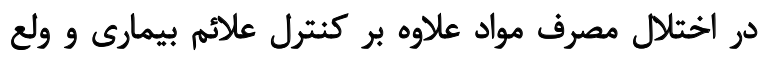

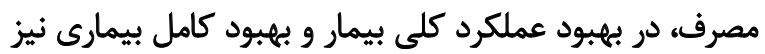

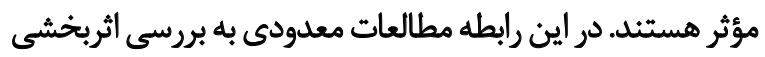

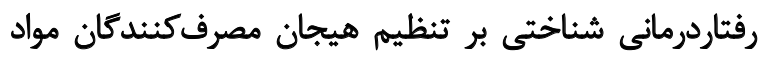

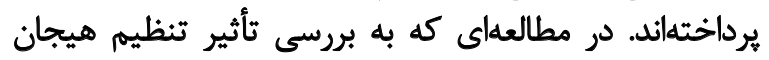

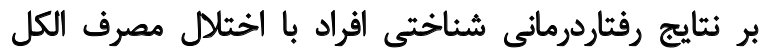

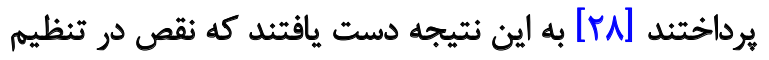

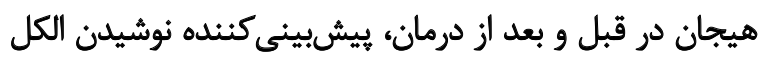

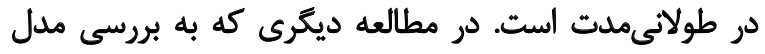

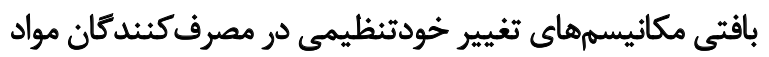

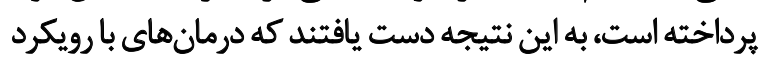

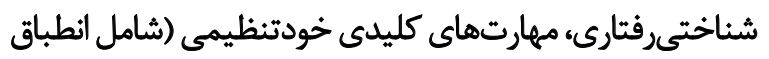

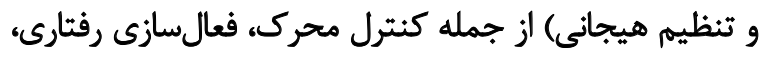

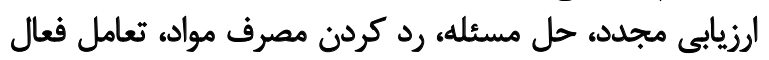

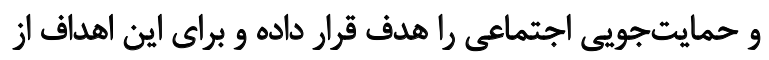

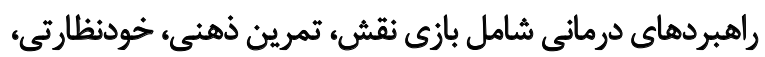

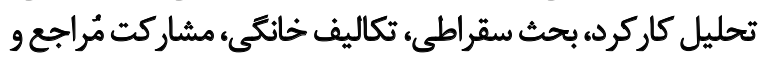

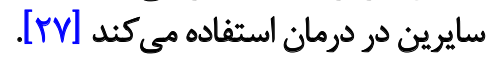

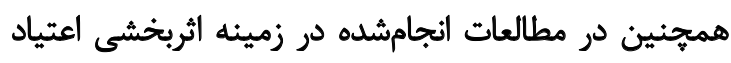

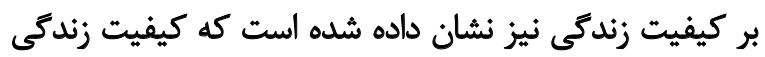

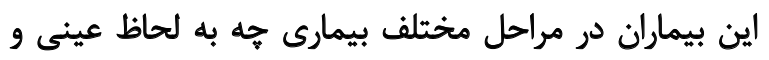

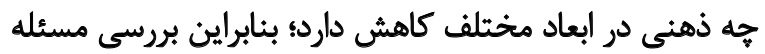

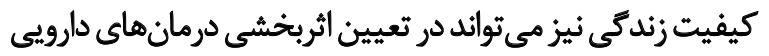

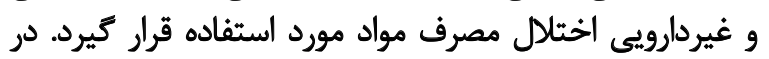

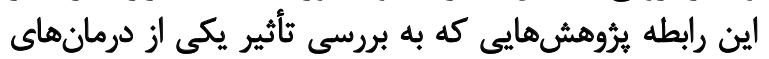

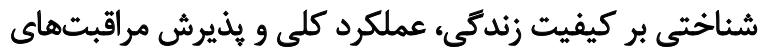

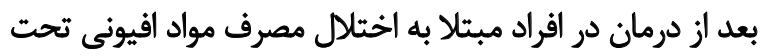

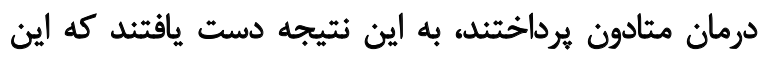

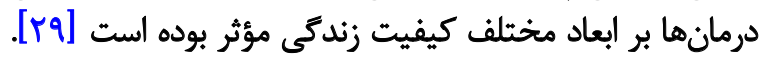

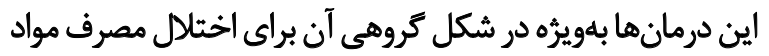

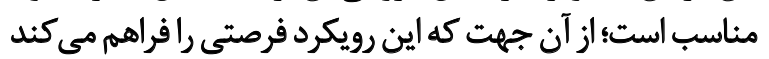

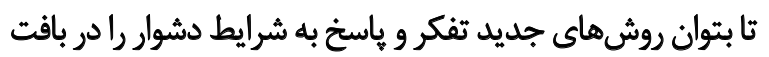

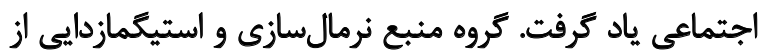

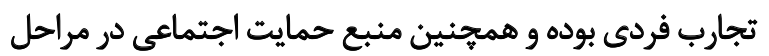

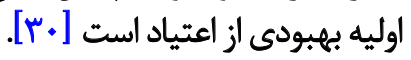
بنابراين با توجه به كاربرد كسترده درمان نتخهدارنده با متادون

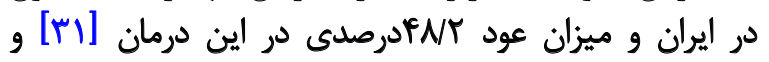

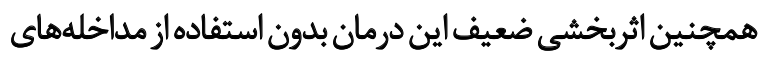

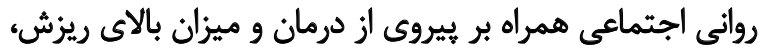

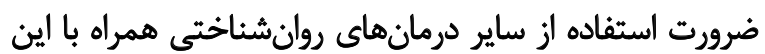


هرسشنامه نظمجويى شناختى هيجان در فرهنَّ ايرانى توسط

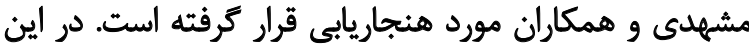

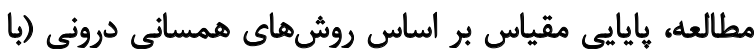

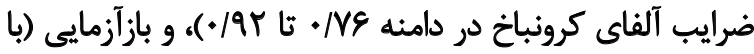

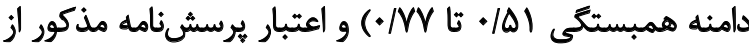
طريق تحليل مؤلفههاى اصلى با استفاده از خرخش وارئ واريماكس،

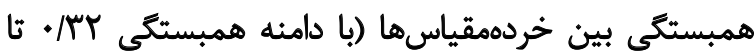
1\&V

\section{مقياس كيفيت زندكى وضعيت جسمانىروانى"}

اين يرسشنامه داراي عب سؤال است كه توسط وور و همكاران

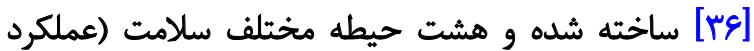
جسمانى، محدوديت نقش جسمانه

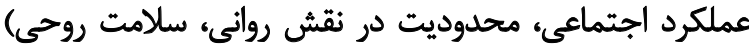

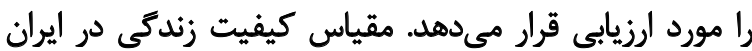

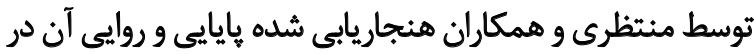

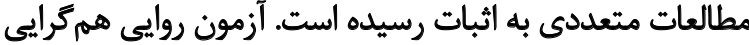

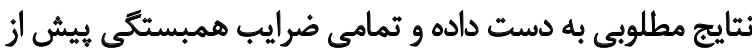

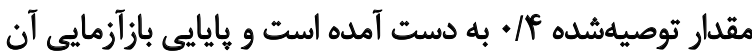

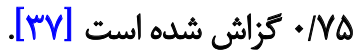

\section{ابزار تردآورى اطلاعات}

\section{"برسشنامه تنظيم شناختى هيجان"}

اين برسشنامه توسط كارنفسكى و همكاران [بF] در كشور

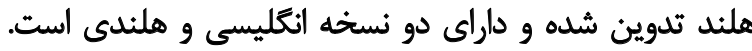

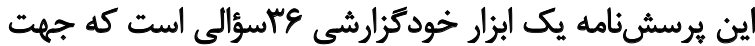

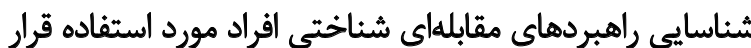

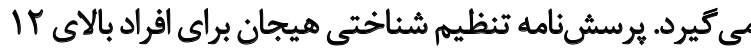

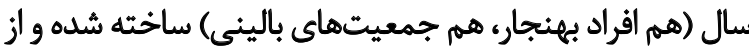

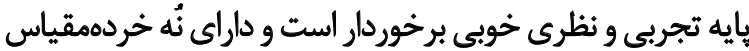

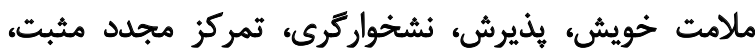

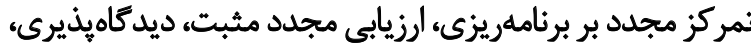

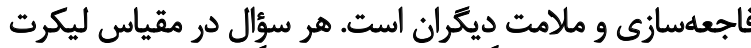

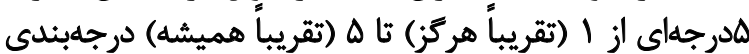

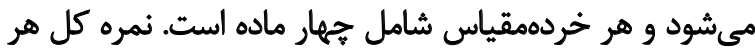

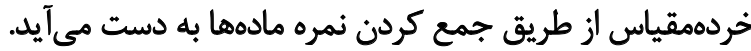

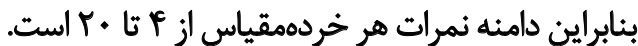

نمرات بالا در هر خردهمقياس، بيانتُ ميزان استفاده بالا از راهبرد در مقابله با وقايع استرسزا و منفى است. نسخه فارسي

11. Cognitive Emotion Regulation Questionnaire (CERQ)

اجراى ييش آزمون. معرفى و آشنايى اعضاى كروه، أموزش مبانى اوليه موسيقى و نتخوانى در سازهاى كوبهاى با مشاركت اعضا، ترغيب اعضا جهت فعاليت كروهى

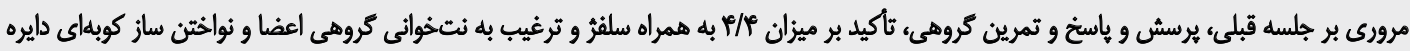

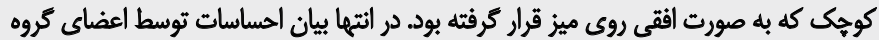

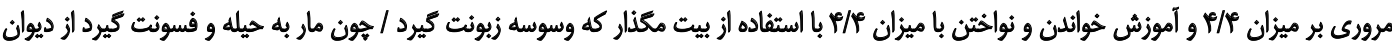

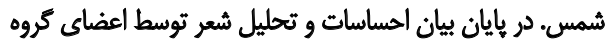

pou

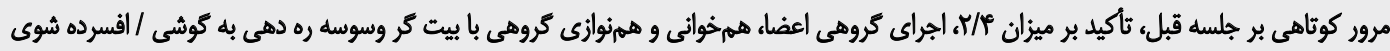

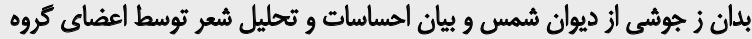

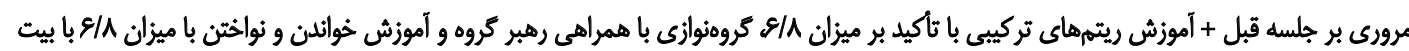

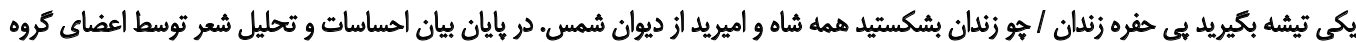

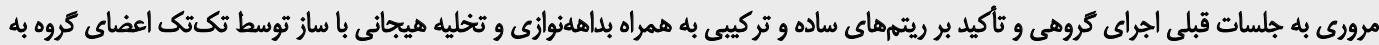

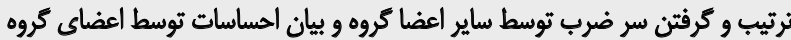

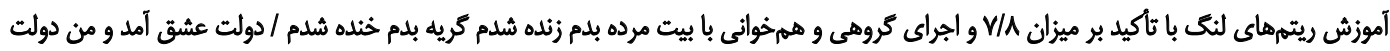

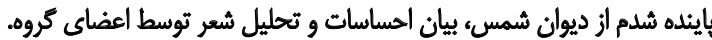


جدول ז. محتواى جلسات آموزش درمان شناختي رفتّارى

\begin{tabular}{|c|c|}
\hline اهم اقدامات صورت كَرفته & جلسه \\
\hline 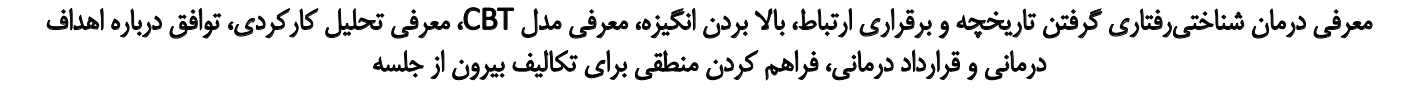 & اول \\
\hline مقابله با ميل به مصرف درك ميل، توصيف ميل، شناسايع بر انكيزان يا تريكرها، اجتناب از نشائهها، مقابله با ميل & دوم \\
\hline تقويث انكيره و تعهل بيمار نسبت به ترك، روشنسازى و اولويتبئلى اهداف، توجه به دوسوكرايع بيمار در مورد ترك، شناسايى و مقابله با افكار مرتبط با & سوم \\
\hline 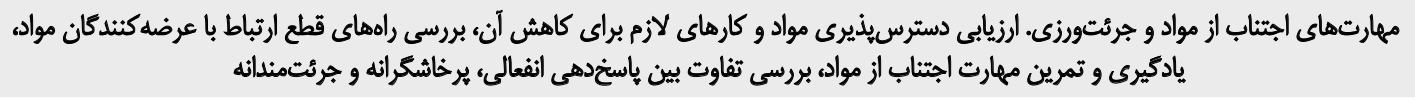 & جهارم \\
\hline 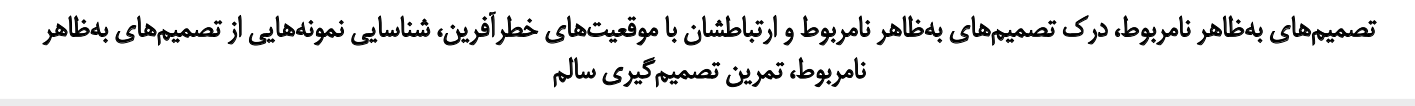 & ينجم \\
\hline 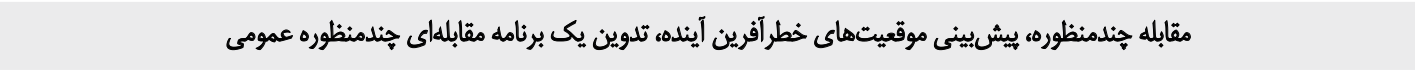 & ششم \\
\hline آموزش مهارت حل هسثله هعرفى كامهاى اساسى حل هسيله تمرين روى مهارتهاي حل مسئل درون جلسه & هُنتم \\
\hline 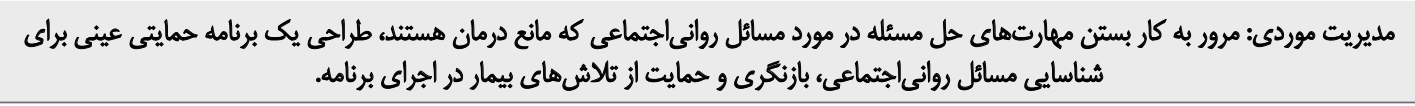 & هشتم \\
\hline
\end{tabular}

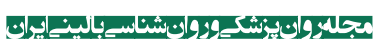

$$
\text { رفتاردرمانى شناختى }
$$

براي زروه دوم، رفتاردرماني شناختي در هشت جلسه و هر

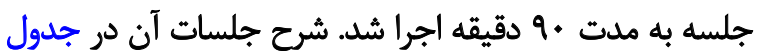

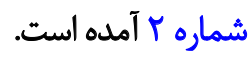

batiols

نتايج نشان داد ميانكين و انحراف معيار سن نمونه يروهش در كروه موسيقى درمانى ب/ه مر

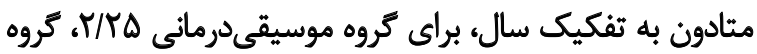

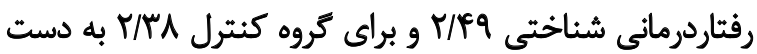

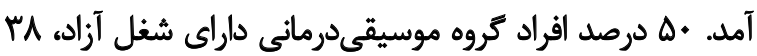
درصد بيكار و || درصد كارمئد بودند. اين ميزان براي كروماني

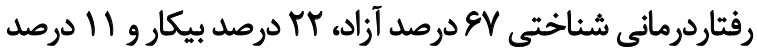

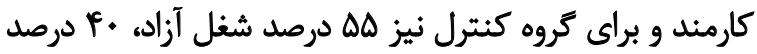

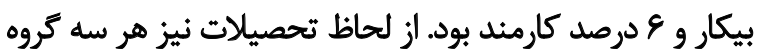

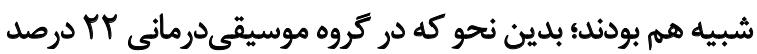

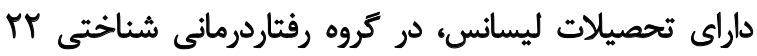

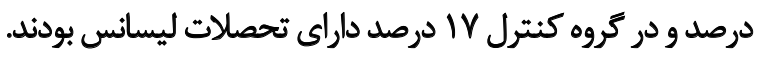

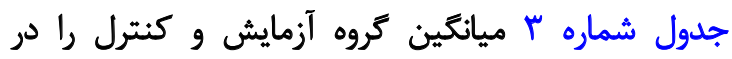

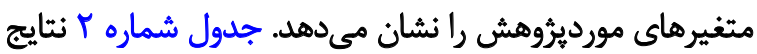

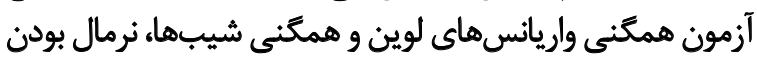

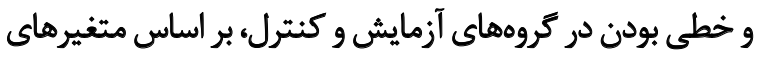
يروهش رانشان مي دهد.

\section{هرسش نامه ولع مصرف"}

اين مقياس يك يرسشنامه fاسؤالي است كه توسط فرانكن

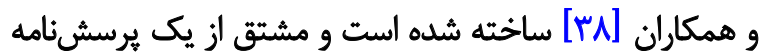

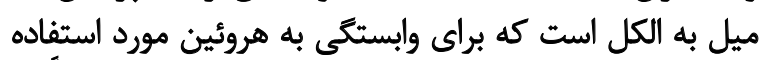

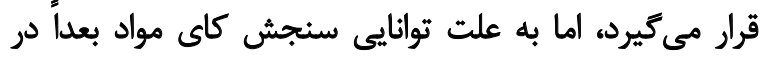

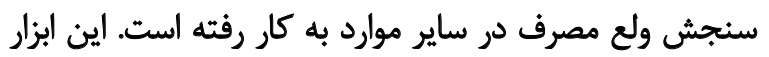
سه زيرمقياس ميل و قصد مصرف مواد، تقويت منفى و كنترل

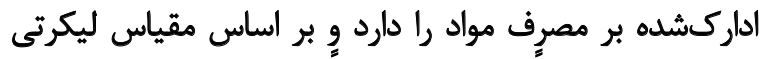

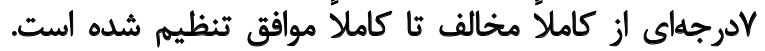

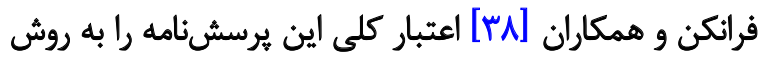

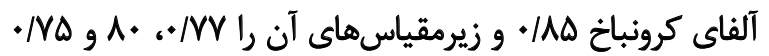

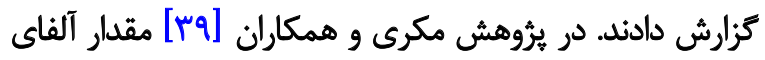

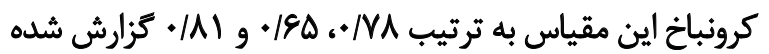

روش مداخله

\section{آموزش موسيقى درمانى}

براي كروه آزمايش هشت جلسه موسيقي درماني به صورت

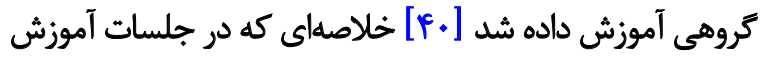

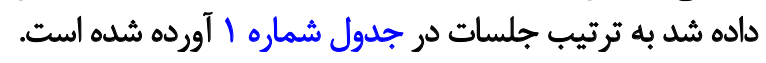

13. Desires For Drug Questionnaire (DDQ) 
جدول ※. ميانكين و انحراف معيار متغيرهاي ثيروهش در كروهاى آزمايش و كنترل

\begin{tabular}{|c|c|c|c|}
\hline \multicolumn{2}{|c|}{ ميانكين I|نحراف معيار } & \multirow{2}{*}{ كروهها } & \multirow{2}{*}{ مثغيرهاى بوروهش } \\
\hline يسآزمون & يريش آزمون & & \\
\hline $\mid M Y / \Delta \cdot \pm E / A Y$ & $1 . F / T V \pm N A 9$ & رفتاردرماني شُناختى & \\
\hline $11 T / \cdot \Delta \pm \| r / r E$ & $1 \cdot r / A r \pm I \Delta / r T$ & هوسيقيدرمائي & كيفيت زندكى \\
\hline $1.9 / \pi Y \pm V / M$ & $1 \cdot N \cdot \Delta \pm V / T Y$ & كنترل & \\
\hline $81 / u \pm V /+r$ & $r A \pm q / i r$ & رفتاردرمانى شُناختى & \\
\hline$\Delta V / \cdots \pm V / r g$ & $\Delta 1 / E I \pm N Q E$ & موسيقىدرمائى & تنظيم مثبتشناختى \\
\hline$\Delta+/ T Y \pm \Delta / \Delta 1$ & $\Delta r / A r \pm 1 . / 8 q$ & كنترل & \\
\hline PV/ATEV/M & $\Delta N / R \pm I T / M$ & رفتاردرماني شناختي & \\
\hline$\Delta \cdot / R Y \pm \xi / T A$ & $\Delta 9 / V T \pm q / \wedge 9$ & هوسيقيدرمانى & تنظيم منفي شناختى \\
\hline$\Delta \& / M \pm P / q P$ & $\Delta \Delta / \Lambda \uparrow \pm N \cdot \varphi$ & كتترل & \\
\hline$P q / W \pm V / \& A$ & $V / \Delta \cdot \pm / \% / . \varphi$ & رفتاردرماني شناختى & \\
\hline$\Delta \Delta / W \pm 1 \cdot / F \Delta$ & $V \Delta / V \cdot \pm 1 r / \cdot r$ & موسيقيدرماني & ولع مصرف \\
\hline$\varepsilon q / T V \pm V / r \Delta$ & 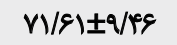 & كنترل & \\
\hline
\end{tabular}

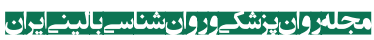

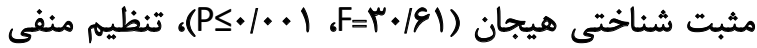

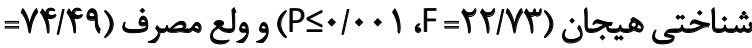

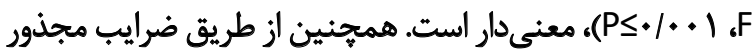
اثتاى تفكيكى مي توان نتيجه كرفت كه مداخله درمانى ثأثير بسيار

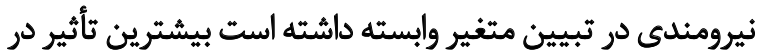

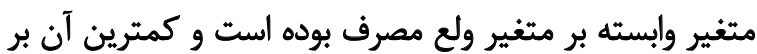

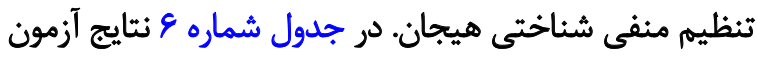

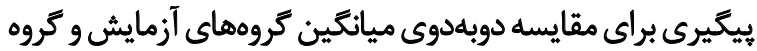

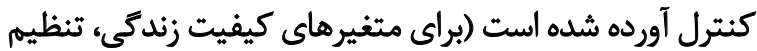

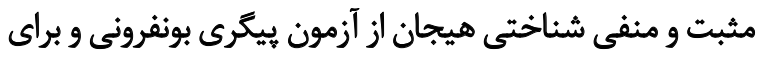

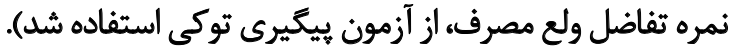
با توجه به اطلاعات جدول شماره عه به دليل كمتر بودن
براى استفاده از تحليل كوواريانس، از آزمون همگنى واريانس و

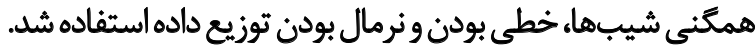

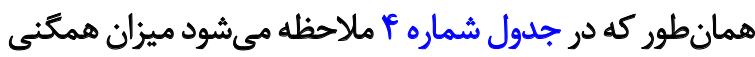

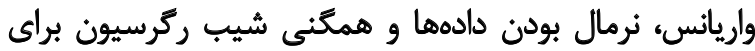

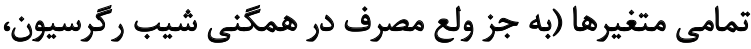

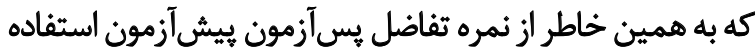

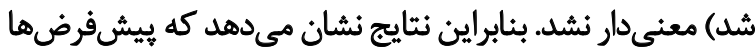

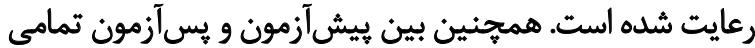

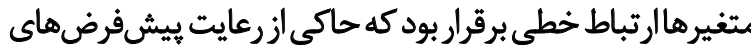
تحليل كوواريانس است. مئس

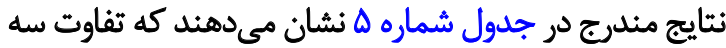

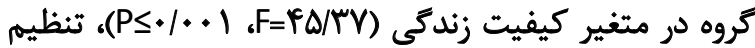

جدول f. نتايج آزمون همكنى واريانسهاى لوين و همكنى شيب در كروهاي آزمايش و كنترل، بر اساس متغيرهاى هيروهش

\begin{tabular}{|c|c|c|c|c|c|c|c|c|}
\hline \multicolumn{2}{|c|}{ ثرمال بودن توزيع داده } & \multicolumn{2}{|c|}{ مُطى بودن } & \multicolumn{2}{|c|}{ همكُنى شيب رئرسيون } & \multicolumn{2}{|c|}{ همكَّنى واريانس } & \multirow{2}{*}{ متغير } \\
\hline $\mathbf{P}$ & K-S & $\mathbf{P}$ & $\mathbf{F}$ & $\mathbf{P}$ & $\mathbf{F}$ & $\mathbf{P}$ & لوين & \\
\hline$+M I M$ & .189 & $+\infty+1$ & $8 / / 4 \Delta$ &.$/ M A$ & I & . & VIr & كيفيت زندكى \\
\hline / /هQT & $\cdot / A V$ & $.1+.1$ & Tr/Ar & $\cdot \pi+$ & $1 / 81$ &.$/ 1 r$ & $r / M I$ & نظيم مثبت شناختى هيجان \\
\hline$V / T+$ &.$/ 18$ & $.1 . F$ & $F / A E$ &.$/ \mathrm{HA}$ & $1 / 1$. &.$/ \mu$ & VTr & نتظيم منفى شناختى هيجان \\
\hline - MeA &.$/ 84$ & $.1 . .1$ & $91 / 88$ & .1 .01 & $r V / . V$ &.$/ 49$ & I/TO & ولع هصرف \\
\hline
\end{tabular}


جدول هـ تحليل كوواريانس يكراهه كيفيت زندگى در دو كروه آزمايش و يك كروه كنترل

\begin{tabular}{|c|c|c|c|c|c|c|}
\hline مجذور اتا & هعنى سطاري & $\mathbf{F}$ & مينظئين مينورات & df & مجنمورات & متغير وابسته \\
\hline$+18 V$ & $\mathrm{P} \leq+1++1$ & $F A / T r$ & $M T+/ M$ & $r$ & mpilife & كيفيت زنلكى \\
\hline.$/ 09$ & $P \leq+1++1$ & $r \cdot|8|$ & $19 \% / A 1$ & $r$ & IVAVIAT & تنظيم مثبت شناختى هيجان \\
\hline - / $\Delta r$ & $P \leq+1++1$ & $r T / N$ & $\Delta A F / M$ & $r$ & IIES/FT & تنظيم مئفى شئاختى هيجان \\
\hline$\cdot M^{e}$ & PS. $1 . .1$ & 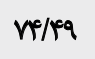 & PAFNAT & $r$ & Veqviea & ولع مصرف (نمره تفاضل يسأزمون ييش آزمون) \\
\hline
\end{tabular}

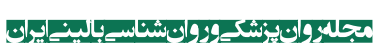

جدول \&. نتايج آزمون يُيكيرى براى مقايسه ميانكينهاى كروهها در كيفيت زندكى در مرحله بسآزمون

\begin{tabular}{|c|c|c|c|c|c|}
\hline هجذور اتا & سطح معنى دارى & خطاى معيار & تفاضل ميانكين & مقايسه & مقياس \\
\hline \multirow{3}{*}{$.18 \mathrm{~V}$} & $P \leq+1 \cdot+1$ & V/AD & $W / \Delta V$ & كروه اورr & \multirow{3}{*}{ كيفيت زندكى } \\
\hline & $P \leq+\mid+.1$ & VAV & IVIFV & كروها اوبr & \\
\hline & $\mathrm{P}=\approx / \cdots \Delta$ & $V / A$. & sl. & كروه r, & \\
\hline \multirow{3}{*}{.109} & $\mathrm{P}=* 1 \cdot \Lambda$ & $r / A$. & NEP & كروه اورr & \multirow{3}{*}{ تنظيم مثبت شناختى هيجان } \\
\hline & $P \leq+/ .+1$ & $r / R T$ & IE/PV & كروه اورr & \\
\hline & $\mathrm{P}=* / \cdot 1$ & $r / 81$ & V/AT & Sروه & \\
\hline \multirow{3}{*}{. $/ A T$} & $P=* 1 \cdot \bullet V$ & $r / \Delta q$ & $-11 / 80$ & كروه اور & \multirow{3}{*}{ تنظيم منفى شناختى هيجان } \\
\hline & $P \leq+/ * 1$ & YTM & $-1 f$ fer & كروها اوبr & \\
\hline & $\mathrm{P}=\star / 89$ & $r / T Q$ & $-T / M$ & Sروه & \\
\hline \multirow{3}{*}{$\cdot M^{\circ}$} & $\mathrm{P}=* / 99$ & $r / r q$ & $-r / \cdot r$ & كروها ورr & \multirow{3}{*}{ 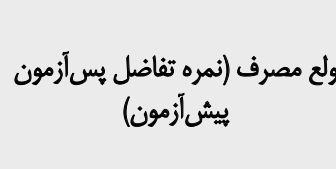 } \\
\hline & $P \leq * 1 * .1$ & $r / m q$ & -re/ry & كروه اورr & \\
\hline & $P \leq * / . .1$ & $r / m q$ & -rfira & كروه & \\
\hline
\end{tabular}

ولع مصرف و افزايش كاهش هيجان هاي شناختى منفى و افزايش

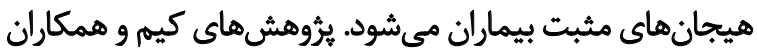

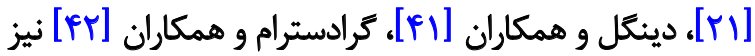
همسو با نتايج اين يثوهش هستند. براى تبيين اين يافته ميثن

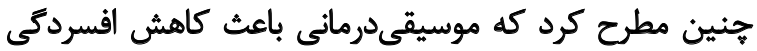

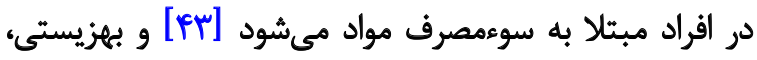
تخليه هيجانى و القاى اميد در بيماران مبتلا به سوءمصرف موف مواد

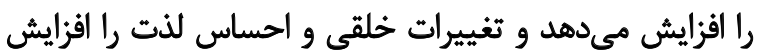
مى ندهد.

يكى از مهمهترين ويزگكى هايى كه موسيقى درمائى دارد اين است

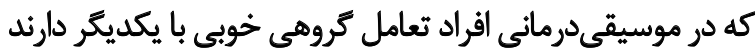

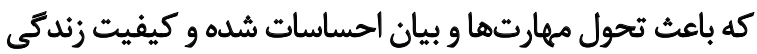

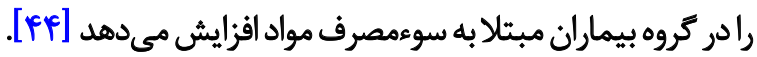

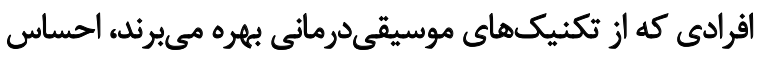

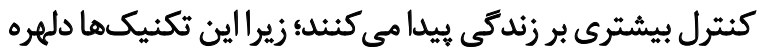

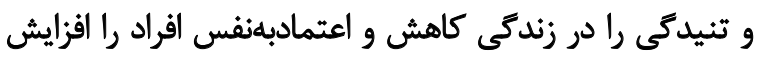

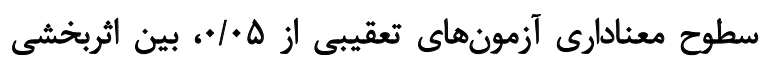

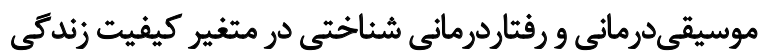

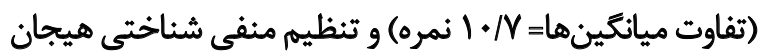

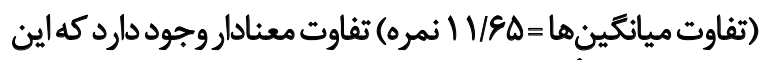

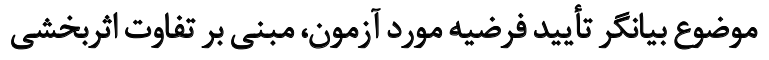

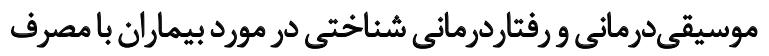

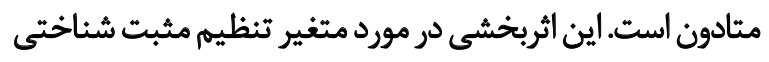

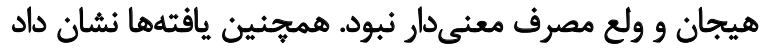

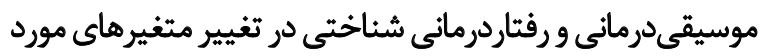
برسى اثربخشى معنى دارى داشتهاند. \&u

هدف از انجام اين ثيروهش مقايسه اثربخشي موسيقي درمانى

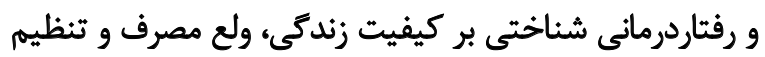

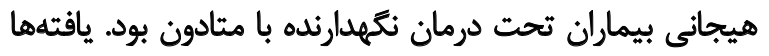

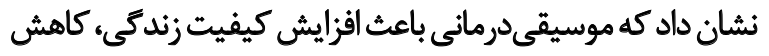




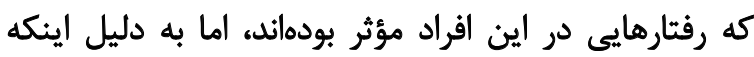

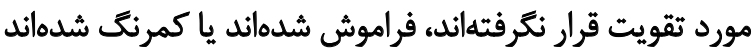

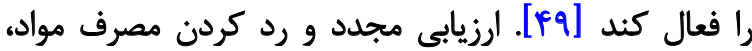

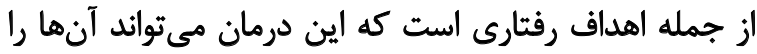

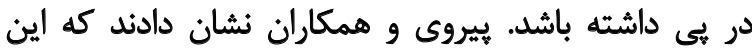

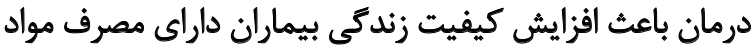

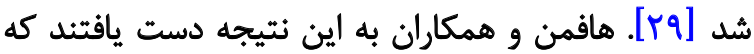

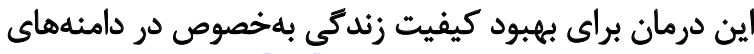

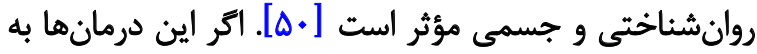

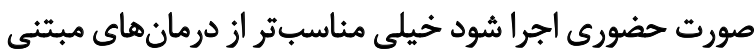

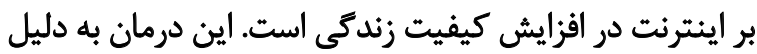

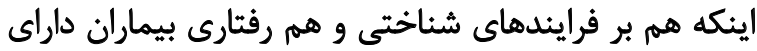

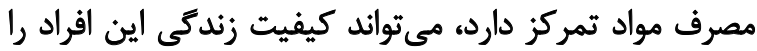

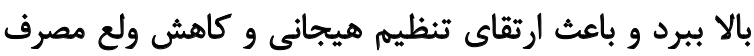

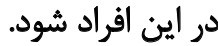

\section{تثيجلكَيرى}

با بررسى مقايسه دو درمان موسيقىدمانى و رفتاردرمانى

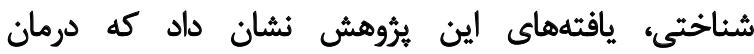

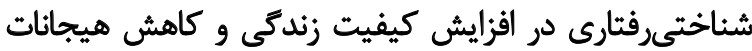

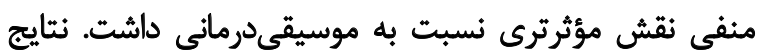

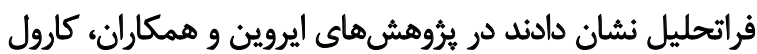

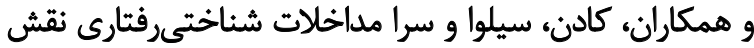

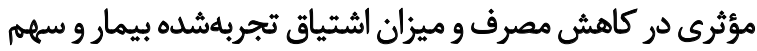

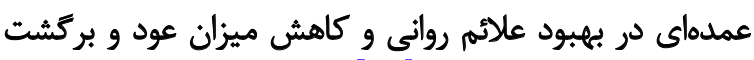

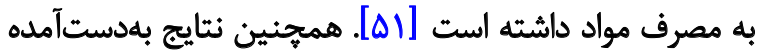

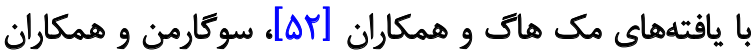

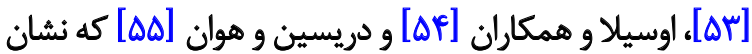

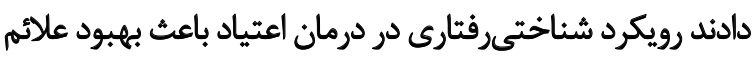

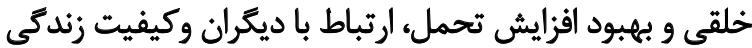
مى شود همسوست.

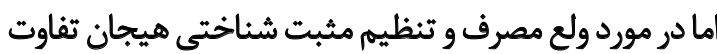

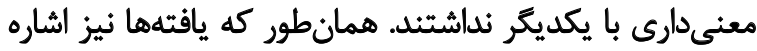

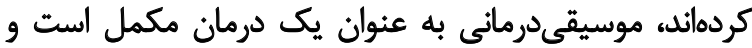

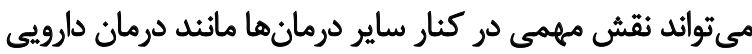

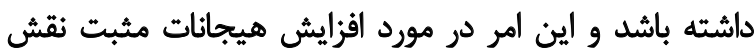

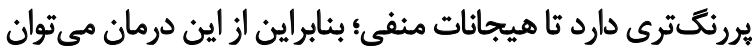

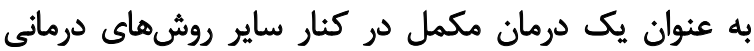

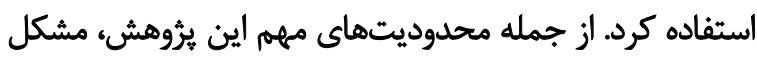

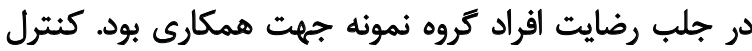

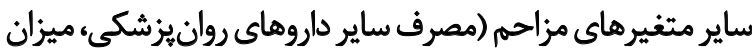

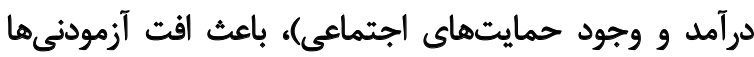

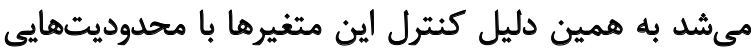

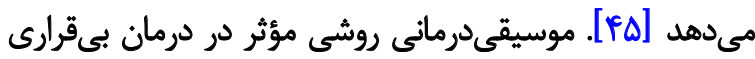

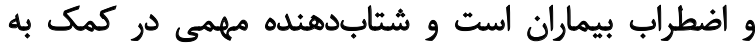

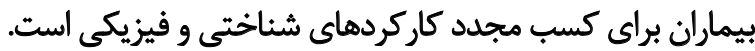

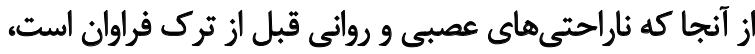

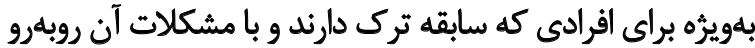

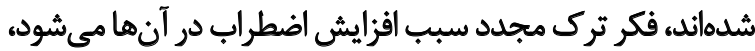

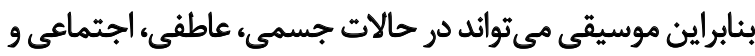

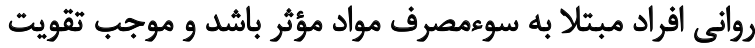

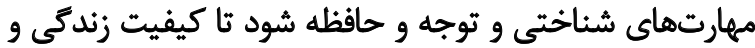
هيجانهاي مثبت آنها افزايش يابد.

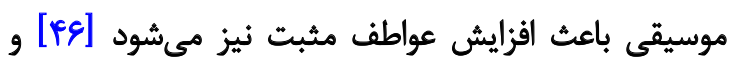

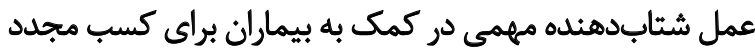

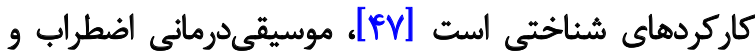

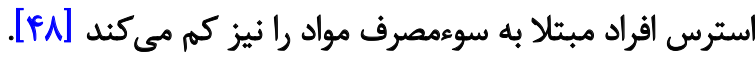

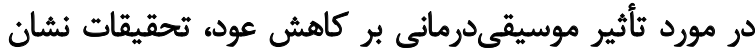

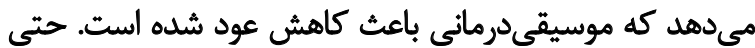

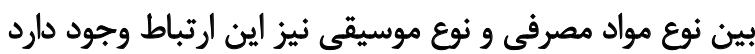

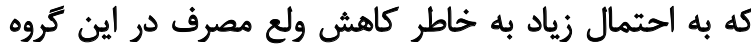

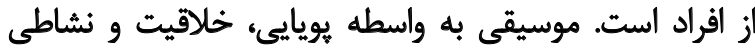

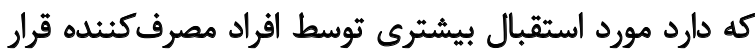

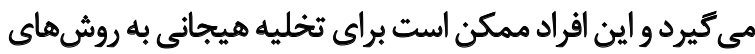

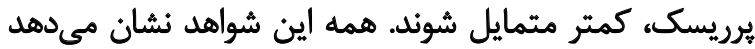

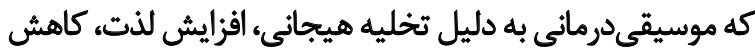

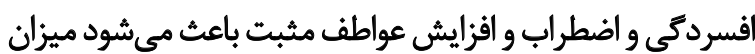

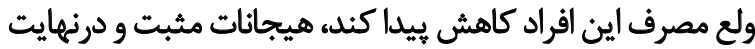

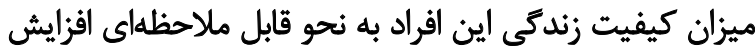

يابِ

همجينين يافتهها نشان داد رفتاردرمانى شناختى باعث افزايش

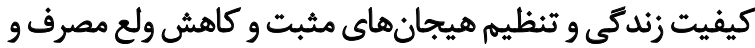

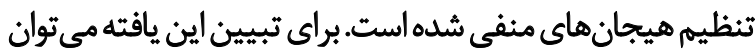

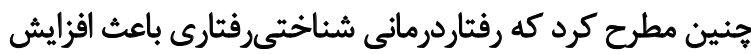

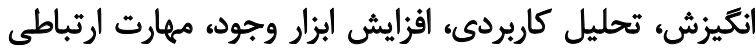

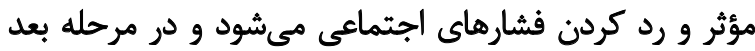

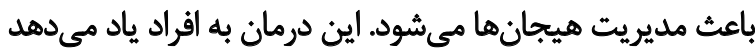

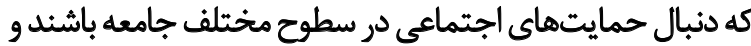

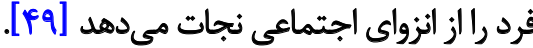

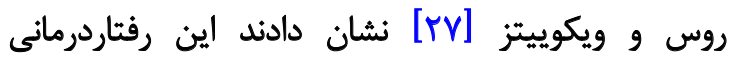

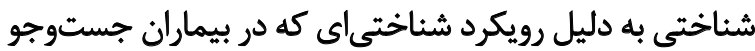

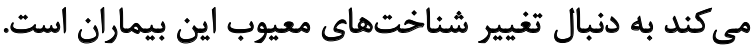

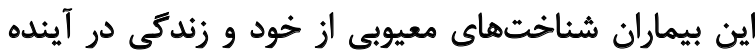

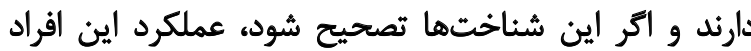

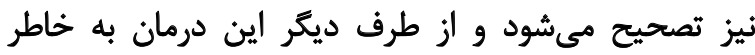
تمركز ويرهاى كه بر فعال سازى رفتارى دارئ دارد، سعى مئ دمان بهند 
مواجه بود. در تعميم نتايج به جنسيت زن، به دليل استفاده از

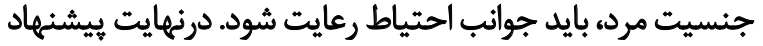

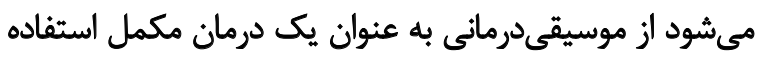

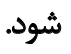
مالاحظات اخلاقي بيروى أز اصول اخلاق يُوهش اين يُوهش در سايت كارآزمايى بالينى كشور با كد

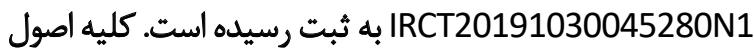

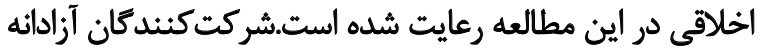

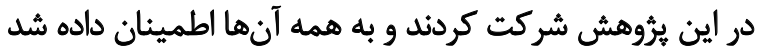
كه اطلاعاتشان محرمانه خواهند ماند كردئ به همه

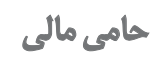

تمام نويسندكان در طراحى، اجرا و نكارش همه بخشهاى ئروهش حاضر مشاركت داشتهاند.

$$
\text { مشاركت نويسند مَان }
$$

اين تحقيق هيج كونه كمك مالى از سازمان هاى تأمين مالى در بخشهاى عمومى ، تجارى يا غيرانتفاعى دريافت نكردي

$$
\text { ت تعارض مثافع }
$$

بنا به اظهار نويسندكان، اين مقاله هيجَّونه تعارض منافعى ندارد. 


\section{References}

[1] Nutt DJ, King LA, Phillips LD. Drug harms in the UK: A multicriteria decision analysis. The Lancet. 2010; 376(9752):1558-65. [DOI:10.1016/S0140-6736(10)61462-6]

[2] Tomás-Rosselló J, Rawson RA, Zarza MJ, Bellows A, Busse A, Saenz E, et al. United Nations Office on Drugs and Crime international network of drug dependence treatment and rehabilitation resource centres: Treatnet. Substance Abuse. 2010; 31(4):251-63. [DOI:10.1080/08897077.2010.514243] [PMID]

[3] Abuse S. Mental Health Services Administration. Results from the. 2013 Jan 10; 2:013.

[4] Jhanjee S. Evidence based psychosocial interventions in substance use. Indian Journal of Psychological Medicine. 2014; 36(2):112-8. [DOI:10.4103/0253-7176.130960] [PMID] [PMCID]

[5] Aletraris L, Paino M, Edmond MB, Roman PM, Bride $\mathrm{BE}$. The use of art and music therapy in substance abuse treatment programs. Journal of Addictions Nursing. 2014; 25(4):190-6. [DOI:10.1097/JAN.0000000000000048] [PMID] [PMCID]

[6] Malchiodi CA, Crenshaw DA, editors. Creative arts and play therapy for attachment problems. New York: Guilford Publications; 2015. https://books.google.com/ books?id=5R80CgAAQBAJ\&dq

[7] Shuman J, Kennedy H, DeWitt P, Edelblute A, Wamboldt MZ. Group music therapy impacts mood states of adolescents in a psychiatric hospital setting. The Arts in Psychotherapy. 2016; 49:50-6. [DOI:10.1016/j.aip.2016.05.014]

[8] Pelletier CL. The effect of music on decreasing arousal due to stress: A meta-analysis. Journal of Music Therapy. 2004; 41(3):192-214. [DOI:10.1093/jmt/41.3.192] [PMID]

[9] Sharma M, Jagdev T. Use of music therapy for enhancing self-esteem among academically stressed adolescents. Pakistan Journal of Psychological Research. 2012; 27(1):53-64. https:// psycnet.apa.org/record/2012-30966-004

[10] Ross S, Cidambi I, Dermatis H, Weinstein J, Ziedonis D, Roth S, et al. Music therapy: A novel motivational approach for dually diagnosed patients. Journal of Addictive Diseases. 2008; 27(1):41-53. [DOI:10.1300/J069v27n01_05] [PMID]

[11] Baker FA, Gleadhill LM, Dingle GA. Music therapy and emotional exploration: Exposing substance abuse clients to the experiences of non-drug-induced emotions. The Arts in Psychotherapy. 2007; 34(4):321-30. [DOI:10.1016/j. aip.2007.04.005]

[12] Gooding LF. The effect of a music therapy social skills training program on improving social competence in children and adolescents with social skills deficits. Journal of Music Therapy. 2011; 48(4):440-62. [DOI:10.1093/jmt/48.4.440] [PMID]

[13] Lin ST, Yang P, Lai CY, Su YY, Yeh YC, Huang MF, et al. Mental health implications of music: Insight from neuroscientific and clinical studies. Harvard Review of Psychiatry. 2011; 19(1):34-46. [DOI:10.3109/10673229.2011.549769] [PMID]

[14] Silverman MJ. Effects of group songwriting on motivation and readiness for treatment on patients in detoxification: A randomized wait-list effectiveness study. Journal of $\mathrm{Mu}$ - sic Therapy. 2012; 49(4):414-29. [DOI:10.1093/jmt/49.4.414] [PMID]

[15] Jones JD. A comparison of songwriting and lyric analysis techniques to evoke emotional change in a single session with people who are chemically dependent. Journal of Music Therapy. 2005; 42(2):94-110. [DOI:10.1093/jmt/42.2.94] [PMID]

[16] Magee WL, Davidson JW. The effect of music therapy on mood states in neurological patients: a pilot study. Journa of Music Therapy. 2002; 39(1):20-9. [DOI:10.1093/jmt/39.1.20] [PMID]

[17] Dougherty KM. Music therapy in the treatment of the alcoholic client. Music Therapy. 1984; 4(1):47-54. [DOI:10.1093/ $\mathrm{mt} / 4.1 .47]$

[18] Rio R. Adults in recovery: A year with members of the choirhouse. Nordic Journal of Music Therapy. 2005; 14(2):107-19. [DOI:10.1080/08098130509478133]

[19] Silverman MJ. Effects of a single lyric analysis intervention on withdrawal and craving with inpatients on a detoxification unit: A cluster-randomized effectiveness study. Substance Use \& Misuse. 2016; 51(2):241-9. [DOI:10.3109/108 26084.2015.1092990] [PMID]

[20] Hohmann L, Bradt J, Stegemann T, Koelsch S. Effects of music therapy and music-based interventions in the treatment of substance use disorders: A systematic review. PloS One. 2017; 12(11):e0187363. [DOI:10.1371/journal. pone.0187363] [PMID] [PMCID]

[21] Dingle GA, Gleadhill L, Baker FA. Can music therapy engage patients in group cognitive behaviour therapy for substance abuse treatment? Drug and Alcohol Review. 2008; 27(2):190-6. [DOI:10.1080/09595230701829371] [PMID]

[22] Stead M, McDermott L, Gordon R, Angus K, Hastings $\mathrm{G}$. A review of the effectiveness of social marketing alcohol, tobacco and substance misuse interventions. National Social Marketing Centre. 2006; Report 3. http:/ / www.asrem. org/corsi_aggiornamento_convegni/comunicazone $\% 20$ per $\% 20$ la\%20slute/Alcol,\%20tabacco\%20ed\%20abuso\%20sostanze\%20 nel\%20Marketing $\% 20$ sociale.PDF

[23] Carroll KM, Ball SA, Nich C, Martino S, Frankforter TL Farentinos $\mathrm{C}$, et al. Motivational interviewing to improve treatment engagement and outcome in individuals seeking treatment for substance abuse: A multisite effectiveness study. Drug and Alcohol Dependence. 2006; 81(3):301-12. [DOI:10.1016/j.drugalcdep.2005.08.002] [PMID] [PMCID]

[24] Feeney GF, Connor JP, Young RM, Tucker J, McPherson A. Improvement in measures of psychological distress amongst amphetamine misusers treated with brief Cognitive-Behavioural Therapy (CBT). Addictive Behaviors. 2006; 31(10):1833-43. [DOI:10.1016/j.addbeh.2005.12.026] [PMID]

[25] Pan S, Jiang H, Du J, Chen H, Li Z, Ling W, et al. Efficacy of cognitive behavioral therapy on opiate use and retention in methadone maintenance treatment in China: A randomised trial. PloS One. 2015; 10(6):e0127598. [DOI:10.1371/ journal.pone.0127598] [PMID] [PMCID]

[26] Raylu N, Oei TP. A cognitive behavioural therapy programme for problem gambling: Therapist manual. London: Routledge; 2010. [DOI:10.4324/9780203850428] 
[27] Roos CR, Bowen S, Witkiewitz K. Baseline patterns of substance use disorder severity and depression and anxiety symptoms moderate the efficacy of mindfulness-based relapse prevention. Journal of Consulting and Clinical Psychology. 2017; 85(11):1041-51. [DOI:10.1037/ccp0000249] [PMID] [PMCID]

[28] Berking M, Margraf M, Ebert D, Wupperman P, Hofmann SG, Junghanns K. Deficits in emotion-regulation skills predict alcohol use during and after cognitive-behavioral therapy for alcohol dependence. Journal of Consulting and Clinical Psychology. 2011; 79(3):307-18. [DOI:10.1037/a0023421] [PMID] [PMCID]

[29] Peyravi H, Alavi SS, Ghanizadeh M. [The effect of compliance with group therapy on quality of life, global function and care after treatment in opium addicted under MMT (Methadone Maintanence Therapy) (Persian)]. Iran Journal of Nursing. 2018; 30(110):45-57. [DOI:10.29252/ijn.30.110.45]

[30] Dingle GA, Cruwys T, Frings D. Social identities as pathways into and out of addiction. Frontiers in Psychology. 2015; 6:1795. [DOI:10.3389/fpsyg.2015.01795] [PMID] [PMCID]

[31] Matin BK, Jalilian F, Alavijeh MM, Ashtarian H, Mahboubi M, Afsar A. Using the PRECEDE model in understanding determinants of quality of life among Iranian male addicts. Global Journal of Health Science. 2014; 6(6):19-27. [DOI:10.5539/gihs.v6n6p19] [PMID] [PMCID]

[32] Ford JD, Steinberg KL, Hawke J, Levine J, Zhang W. Randomized trial comparison of emotion regulation and relational psychotherapies for PTSD with girls involved in delinquency. Journal of Clinical Child \& Adolescent Psychology. 2012; 41(1):27-37. [DOI:10.1080/15374416.2012.63234 3] [PMID]

[33] Willenbring ML. The past and future of research on treatment of alcohol dependence. Alcohol Research \& Health. 2010; 33(1-2):55-63. [PMID] [PMCID]

[34] Garnefski N, Kraaij V. The cognitive emotion regulation questionnaire. European Journal of Psychological Assessment. 2007; 23(3):141-9. [DOI:10.1027/1015-5759.23.3.141]

[35] Mashhadi A, Hasani J, Mirdoraghi F. [Factor structure, reliability and validity of Persian version of the Cognitive Emotion Regulation Questionnaire-children form (CERQKP) (Persian)]. Journal of Fundamentals of Mental Health. 2012; 14(55):246-59. [DOI:10.22038/JFMH.2013.942]

[36] Ware Jr JE, Gandek B. Overview of the SF-36 health survey and the International Quality of Life Assessment (IQOLA) project. Journal of Clinical Epidemiology. 1998; 51(11):903-12. [DOI:10.1016/S0895-4356(98)00081-X]

[37] Montazeri A, Goshtasebi A, Vahdaninia M, Gandek B. The Short Form Health Survey (SF-36): Translation and validation study of the Iranian version. Quality of Life Research 2005; 14(3):875-82. [DOI:10.1007/s11136-004-1014-5] [PMID]

[38] Franken IHA, Hendriks VM, van den Brink W. Initial validation of two opiate craving questionnaires: The obsessive compulsive drug use scale and the desires for drug questionnaire. Addictive Behaviors. 2002; 27(5):675-85. [DOI:10.1016/ S0306-4603(01)00201-5]

[39] Hassani-Abharian P, Mokri A, Ganjgahi H, Oghabian MA, Ekhtiari H. Validation for Persian versions of "desire for drug questionnaire" and "obsessive compulsive drug use scale" in heroin dependents. Archives of Iranian Medicine. 2016; 19(9):659-65. [PMID]

[40] Unkefer RF, Thaut M, editors. Music therapy in the treatment of adults with mental disorders: Theoretical bases and clinical interventions. New York: Schirmer Books; 1990. https:// books.google.fr/books?hl=fr\&id=iEQJAQAAMAAJ\&dq

[41] Kim KJ, Lee SN, Lee BH. Music therapy inhibits morphine-seeking behavior via GABA receptor and attenuates anxiety-like behavior induced by extinction from chronic morphine use. Neuroscience Letters. 2018; 674:81-7. [DOI:10.1016/j.neulet.2018.03.035] [PMID]

[42] Gardstrom SC, Klemm A, Murphy KM. Women's perceptions of the usefulness of group music therapy in addictions recovery. Nordic Journal of Music Therapy. 2017; 26(4):33858. [DOI:10.1080/08098131.2016.1239649]

[43] Streeter E, Davies MEP, Reiss JD, Hunt A, Caley R, Roberts C. Computer aided music therapy evaluation: Testing the Music Therapy Logbook prototype 1 system. The Arts in Psychotherapy. 2012; 39(1):1-10. [DOI:10.1016/j. aip.2011.11.004

[44] Ghetti C, Chen XJ, Fachner J, Gold C. Music therapy for people with substance use disorders. Cochrane Database of Systematic Reviews. 2017; 2017(3):CD012576. [DOI:10.1002/14651858.CD012576] [PMCID]

[45] Choi AN, Lee MS, Lee JS. Group music intervention reduces aggression and improves self-esteem in children with highly aggressive behavior: A pilot controlled trial. Evidence-Based Complementary and Alternative Medicine. 2010; 7(2):213-7. [DOI:10.1093/ecam/nem182] [PMID] [PMCID]

[46] Jasemi Zergani E, Naderi F. The effectiveness of music on quality of life and anxiety symptoms in the post traumatic stress disorder in Bustan Hospital of Ahvaz City. Review of European Studies. 2016; 8(3):237-43. [DOI:10.5539/res. v8n3p237]

[47] Dillard LM. Musical countertransference experiences of music therapists: A phenomenological study. The Arts in Psychotherapy. 2006; 33(3):208-17. [DOI:10.1016/j. aip.2006.01.002]

[48] Silverman MJ. Effects of music therapy on psychiatric patients' proactive coping skills: Two pilot studies. The Arts in Psychotherapy. 2011; 38(2):125-9. [DOI:10.1016/j. aip.2011.02.004]

[49] Coates JM, Gullo MJ, Feeney GF, Young RM, Connor JP. A randomized trial of personalized cognitive-behavior therapy for alcohol use disorder in a public health clinic. Frontiers in Psychiatry. 2018; 9:297. [DOI:10.3389/fpsyt.2018.00297] [PMID] [PMCID]

[50] Hoffman CJ, Ersser SJ, Hopkinson JB, Nicholls PG, Harrington JE, Thomas PW. Effectiveness of mindfulness-based stress reduction in mood, breast-and endocrine-related quality of life, and well-being in stage 0 to III breast cancer: a randomized, controlled trial. Journal of Clinical Oncology. 2012; 30(12):1335-42. [DOI:10.1200/JCO.2010.34.0331] [PMID]

[51] Miller WR, Sorensen JL, Selzer JA, Brigham GS. Disseminating evidence-based practices in substance abuse treatment: A review with suggestions. Journal of Substance Abuse Treatment. 2006; 31(1):25-39. [DOI:10.1016/j. jsat.2006.03.005] [PMID] 
[52] McHugh RK, Hearon BA, Otto MW. Cognitive behavioral therapy for substance use disorders. Psychiatric Clinics of North America. 2010; 33(3):511-25. [DOI:10.1016/j. psc.2010.04.012] [PMID] [PMCID]

[53] Sugarman DE, Nich C, Carroll KM. Coping strategy use following computerized cognitive-behavioral therapy for substance use disorders. Psychology of Addictive Behaviors. 2010; 24(4):689-95. [DOI:10.1037/a0021584] [PMID] [PMCID]

[54] Osilla KC, Hepner KA, Muñoz RF, Woo S, Watkins K. Developing an integrated treatment for substance use and depression using cognitive-behavioral therapy. Journal of Substance Abuse Treatment. 2009; 37(4):412-20. [DOI:10.1016/j. jsat.2009.04.006] [PMID] [PMCID]

[55] Driessen E, Hollon SD. Motivational interviewing from a cognitive behavioral perspective. Cognitive and Behavioral Practice. 2011; 18(1):70-3. [DOI:10.1016/j.cbpra.2010.02.007] 
This Page Intentionally Left Blank 